\title{
Umbilic Lines in Orientational Order
}

\author{
Thomas Machon and Gareth P. Alexander \\ Department of Physics and Centre for Complexity Science, University of Warwick, \\ Coventry CV4 7AL, United Kingdom
}

(Received 6 July 2015; revised manuscript received 23 November 2015; published 28 March 2016)

Three-dimensional orientational order in systems whose ground states possess nonzero gradients typically exhibits linelike structures or defects: $\lambda$ lines in cholesterics or Skyrmion tubes in ferromagnets, for example. Here, we show that such lines can be identified as a set of natural geometric singularities in a unit vector field, the generalization of the umbilic points of a surface. We characterize these lines in terms of the natural vector bundles that the order defines and show that they give a way to localize and identify Skyrmion distortions in chiral materials - in particular, that they supply a natural representative of the Poincare dual of the cocycle describing the topology. Their global structure leads to the definition of a self-linking number and helicity integral which relates the linking of umbilic lines to the Hopf invariant of the texture.

DOI: 10.1103/PhysRevX.6.011033

Subject Areas: Condensed Matter Physics, Interdisciplinary Physics, Soft Matter

\section{INTRODUCTION}

Three-dimensional systems described by orientational order, and more generally vector fields, are often seen to contain linelike geometrical features. For example, in nematic liquid crystals as escaped disclinations and thick lines [1,2], cholesteric liquid crystals as $\lambda$ lines or dislocations [3-7], and as double twist cylinders in blue phases $[8,9]$, in superfluids they arise as the cores of vortices [10-13], and they can be identified in the Skyrmion [14] textures of ferromagnets [15-22], Bose-Einstein condensates [23-28], and liquid crystals $[3,29-33]$. In this paper we show that a large number of these linelike structures can be identified with a set of natural geometric singularities in a vector field, which we call umbilic lines. These lines, which correspond to local minima of several free energies and Hamiltonians, attain topological significance through their correspondence to Skyrmions, giving a natural relationship between the geometric and topological features of orientational order. Typically identified in an ad hoc fashion, our approach gives a principled description and rigorous definition of these lines that can be applied to any system. In addition to giving a more thorough understanding of existing experimental systems such as isolated and periodic Skyrmions, oily streaks, confined chiral solitons $[3,30]$, the blue phases $[8,9,34]$, and colloidal dispersions [35], our work motivates

Published by the American Physical Society under the terms of the Creative Commons Attribution 3.0 License. Further distribution of this work must maintain attribution to the author(s) and the published article's title, journal citation, and DOI. the exploration of closed umbilic loops as examples of topologically nontrivial textures.

Skyrmions in chiral magnets are often described as magnetic whirls that encode a topological winding. In many cases they are easy to recognize when you see them, for instance, in the direct real space images produced by Lorentz transmission electron microscopy [19]. The same can be said for double twist cylinders in blue phases or the $\lambda$ lines of cholesterics, the latter readily identifiable using fluorescence confocal polarizing microscopy [7]; however, it is telling that there is no general theoretical construction for locating the axes of double twist cylinders in an arbitrary director field, or $Q$ tensor, nor an established fully general definition of the pitch axis in a cholesteric [36]. The theoretical identification of Skyrmions is in terms of a conserved topological charge; it is a global property of the entire system, for instance, given by the integral of a density over the entire domain [see Eq. (10)]. As a global quantity, this does not identify local properties of the texture, such as the real space location of individual Skyrmions. Ad hoc rules may be given, such as taking the maxima of the charge density [integrand in Eq. (10)], or for quasi-two-dimensional samples places where the magnetization is parallel to a preferred direction, such as that of an applied magnetic field. An influential early paper of Belavin and Polyakov [37] gives local positional information through the zeros and poles of a meromorphic function. This description is for a two-dimensional domain and a quadratic gradient energy function without any form of spin-orbit coupling usually associated with the stability of Skyrmions. As we explain later, the description they provide is very special-it corresponds to a totally umbilic configuration-and so does not represent a generic 
situation. For arbitrary three-dimensional textures there is no general theoretical formalism for identifying localized linelike structures in liquid crystals, magnetic materials, or any other system with orientational order. This may be contrasted with an analogous situation in optics where linelike degeneracies in the electromagnetic field known as $C$ lines (points where the polarization is circular rather than elliptical) can be both observed experimentally and identified, in full generality, from theoretical expressions given in terms of the electric and magnetic fields [38-44]. We provide a fully generic theoretical identification of linelike features in orientational order, applicable to nematic and cholesteric liquid crystals and to Skyrmion textures in chiral magnets, spin configurations of Bose-Einstein condensates, superfluids, and electronic states.

The umbilics we define are natural geometrical degeneracies of the gradients of the orientational order that convey its topology since they are Poincaré dual to twice the Euler class of the 2-plane bundle of directions that are everywhere orthogonal to the local director. By an application of the Gauss-Bonnet-Chern theorem, the flux of umbilics through any surface is identified with the Skyrmion charge. In this way umbilics provide a natural localization of Skyrmions in two-dimensional systems to collections of points, the centers of the vortex distortions with which Skyrmions are often associated, as well as a robust method for the identification of more subtle excitations, such as merons [45] or "halfSkyrmions." In three dimensions umbilic lines provide an identification of structures such as escaped defect lines and Skyrmion tubes, elucidating many systems, particularly those containing closed umbilic loops such as torons [30] and escaped defect lines associated with colloidal dispersions [35], where our formalism shows that the presence of these defect lines is a consequence of symmetries of the system. The relationship given by the Euler class also describes how umbilic lines interact with the disclinations found in nematic order, which allows for a greater variety of three-dimensional textures including knots, a focus of activity across several disciplines [46-51], which can then be entangled by Skyrmions [52] and, consequently, umbilic lines. Theoretically, the extended nature of closed umbilic loops entails additional global properties that we describe in terms of self-linking and linking numbers. An analog of helicity connects these to the Hopf charge in the system in a manner reminiscent of that for fluid flows [53-55]. To our knowledge, these global properties afforded to closed umbilic loops have not yet been explored in experimental systems. However, with the increasing precision that topological structures are being manipulated in experimental systems and with the potential for the development of structured metamaterials [56-58], fluidic photonics [59], and ultralow current spintronics [60-62], we hope that they will provide motivation for future developments.

The umbilics we describe are generic traits, exhibited by any vector or line field (or, indeed, $Q$ tensor), and are closely analogous to several other structural degeneracies, for instance, the lines of circular polarization ( $C$ lines) in a generic electromagnetic field [38-41] and the umbilic points of surfaces [63,64] and random fields [42-44, 65-69], from which the terminology derives and of which they are a generalization. To say more, closed orientable surfaces can be classified topologically by their Euler characteristic. The famous Gauss-Bonnet theorem expresses this integer as an integral of the Gaussian curvature over the surface (divided by $2 \pi$ ), creating a link between curvature and topology. There is an exactly dual connection in terms of the principal curvatures. Degeneracies in the principal curvatures are called umbilic points, to each of which one may associate a half-integer, the winding number of the principal curvature directions about the degeneracy. The sum of the winding numbers of all the umbilics is again the Euler characteristic. The relationship between this way of representing it and the Gauss-Bonnet theorem is an instance of Poincaré duality. Degeneracies in geometrical physical properties are commonplace; their relevance and significance for the description of global or structural aspects of physical behavior, and the identification of generic traits, has been cemented since Berry's influential paper on the geometrical phase in quantum mechanics [70,71], and the same Berry phases control aspects of the response in magnetic Skyrmions [72-74].

The umbilics we describe here acquire extra significance in materials where the ground state has non-zero gradients. In these materials the vortex configurations surrounding umbilics often correspond to local minima of the free energy. The most common of these systems are chiral, so for concreteness we consider ordered materials described by a unit magnitude vector field $\mathbf{n}$, which we call the director, and whose free energy has a form similar to

$$
F=\frac{K}{2} \int_{\mathbb{R}^{3}}\left[|\nabla \mathbf{n}|^{2}+2 q_{0} \mathbf{n} \cdot \boldsymbol{\nabla} \times \mathbf{n}\right] d^{3} x,
$$

where $K$ is an elastic constant and $q_{0}$ is the strength of the chirality, or magnitude of spin-orbit coupling. This is the Frank free energy [75] for a bulk cholesteric under the one elastic constant approximation and also the Hamiltonian for elastic distortions in a chiral ferromagnet with a Dzyaloshinskii-Moriya interaction [76,77]. Of additional note is a connection of our description with recent work of Efrati and Irvine [78] on chiral shapes and structures. In their work they introduced a chirality pseudotensor to characterize the direction of twisting and sense of chirality of various materials. We demonstrate a general connection with the shape operator for a surface or vector field, from which their chirality pseudotensor can be derived, and show that natural benefits are gained by considering both operators. Finally, we note that analogous geometrical structures to the umbilics we describe have been discussed 
by Thorndike et al. [79], Čopar et al. [80], and Beller et al. [36], although not with the topological aspect that we give here.

The contents of this paper may be summarized as follows. The next section gives a complete overview of the properties of umbilics, their local identification and structure, through a local geometric analysis of the derivatives of the orientational order, and summarizes their relation to current experiments on Skyrmions, $\lambda$ lines, monopoles, torons, and other topological structures in vector fields. The following section outlines how to express this local analysis in terms of a $Q$ tensor so that it can be applied also to line fields. This is largely done by direct algebraic constructions with a focus on the application to blue phases, with the technical details largely omitted. It is hoped that these two sections will present all of the core results of this paper in nontechnical form, using only standard vector calculus, and illustrate the many applications, experimental connections, and unity of concepts across several disciplines. The remainder of the paper gives a more technical account of umbilics, establishing their global topological significance. First, we give a definition in terms of vector bundles and establish the general connection with Skyrmions using the Gauss-BonnetChern theorem. This is followed by a discussion of the variation of the local profile of an umbilic along its contour length and of the topology of the space of such local profiles. The final section gives the global extension to the case of umbilic loops, which we see as having the greatest potential for future developments, both theoretical and experimental.

\section{LOCAL STRUCTURE OF ORIENTATIONAL ORDER AND UMBILIC LINES}

Three-dimensional orientational order in a material can be given the following generic characterization. At every point in space there is a local symmetry corresponding to rotations which preserve $\mathbf{n}$. Under the action of this rotation, the gradient tensor $\nabla \mathbf{n}$ decomposes into irreducible representations as

$$
\begin{aligned}
\nabla_{i} n_{j}= & n_{i} n_{k} \partial_{k} n_{j}+\frac{\boldsymbol{\nabla} \cdot \mathbf{n}}{2}\left(\delta_{i j}-n_{i} n_{j}\right) \\
& +\frac{\mathbf{n} \cdot \boldsymbol{\nabla} \times \mathbf{n}}{2} \epsilon_{i j k} n_{k}+\Delta_{i j}
\end{aligned}
$$

The first of these corresponds to the gradients along $\mathbf{n}$, known as bend distortions. The remaining parts correspond to gradients along directions orthogonal to $\mathbf{n}$, the first two of which are isotropic and comprise splay and twist distortions. The final component, $\Delta_{i j}$, corresponds to the anisotropic orthogonal gradients of $\mathbf{n}$, and contains saddlesplay distortions. We assume for now that $\mathbf{n}$ is a vector, rather than a director. The local description of line fields is exactly the same, as can be seen by introducing a local orientation for the director field, and we defer a more thorough discussion until later. The decomposition in Eq. (2) carries over into the energy through expressions for the norm $|\nabla \mathbf{n}|^{2}$, which we write in the well-known form [75]

$$
\begin{aligned}
|\nabla \mathbf{n}|^{2}= & \frac{1}{2}(\boldsymbol{\nabla} \cdot \mathbf{n})^{2}+\frac{1}{2}(\mathbf{n} \cdot \boldsymbol{\nabla} \times \mathbf{n})^{2}+((\mathbf{n} \cdot \boldsymbol{\nabla}) \mathbf{n})^{2} \\
& +2|\Delta|^{2},
\end{aligned}
$$

where (the choice of normalization for the norm of $\Delta$ is motivated by subsequent considerations)

$$
\begin{aligned}
|\Delta|^{2}= & \left(\frac{\boldsymbol{\nabla} \cdot \mathbf{n}}{2}\right)^{2}+\left(\frac{\mathbf{n} \cdot \boldsymbol{\nabla} \times \mathbf{n}}{2}\right)^{2} \\
& -\frac{1}{2} \boldsymbol{\nabla} \cdot[\mathbf{n}(\boldsymbol{\nabla} \cdot \mathbf{n})-(\mathbf{n} \cdot \boldsymbol{\nabla}) \mathbf{n}] .
\end{aligned}
$$

Local minima of free energies such as Eq. (1) can then be expressed in terms of this decomposition. In particular, if $((\mathbf{n} \cdot \boldsymbol{\nabla}) \mathbf{n})^{2}=\boldsymbol{\nabla} \cdot \mathbf{n}=|\Delta|^{2}=0$, then the local energy density of Eq. (1) is given by

$$
\frac{K}{2}\left(\frac{1}{2}(\mathbf{n} \cdot \boldsymbol{\nabla} \times \mathbf{n})^{2}+2 q_{0} \mathbf{n} \cdot \boldsymbol{\nabla} \times \mathbf{n}\right)
$$

and achieves an optimal lower bound of $-K q_{0}^{2}$ for $\mathbf{n} \cdot \boldsymbol{\nabla} \times \mathbf{n}=-2 q_{0}[8,9]$. The local minima of Eq. (1) are thus characterized by having zero splay, zero bend, twist equal to $-2 q_{0}$, and $|\Delta|^{2}=0$. We define points where $|\Delta|^{2}=0$ as umbilics of the orientational order; they, therefore, correspond to regions of the material where the orthogonal gradients of $\mathbf{n}$ are isotropic. In computational settings, umbilic lines can be identified by plotting isosurfaces of $|\Delta|^{2}$, which are shown as blue tubes throughout this paper. In some cases there is significant variation in the size of the tubes. In part, this reflects the structure of the orientational order around the umbilic, although some features, such as the shrinking to zero size, are artifacts of the finite difference schemes used in the computer simulations and do not represent any physical phenomena.

If $\mathbf{d}_{1}, \mathbf{d}_{2}$ are arbitrary unit vectors orthogonal to $\mathbf{n}$, such that $\left\{\mathbf{d}_{1}, \mathbf{d}_{2}, \mathbf{n}\right\}$ form a right-handed set, then the orthogonal gradients can be expressed in this local basis as a $2 \times 2$ real matrix with the decomposition

$$
\begin{aligned}
\nabla_{\perp} \mathbf{n}= & \frac{\boldsymbol{\nabla} \cdot \mathbf{n}}{2}\left[\begin{array}{ll}
1 & 0 \\
0 & 1
\end{array}\right]+\frac{\mathbf{n} \cdot \boldsymbol{\nabla} \times \mathbf{n}}{2}\left[\begin{array}{cc}
0 & -1 \\
1 & 0
\end{array}\right] \\
& +\left[\begin{array}{cc}
\Delta_{1} & \Delta_{2} \\
\Delta_{2} & -\Delta_{1}
\end{array}\right]
\end{aligned}
$$

where 
$\Delta_{1}=\frac{1}{2}\left(\partial_{1} n_{1}-\partial_{2} n_{2}\right), \quad \Delta_{2}=\frac{1}{2}\left(\partial_{1} n_{2}+\partial_{2} n_{1}\right)$.

The vanishing of $\Delta$ is then analogous to the CauchyRiemann equations. Indeed, director configurations whose stereographic projection is a meromorphic function have $\Delta_{1}=\Delta_{2}=0$ everywhere; they are totally umbilic. As shown by Belavin and Polyakov [37], these arise naturally as the minimizers of Eq. (1) with $q_{0}=0$ when the domain is two dimensional. However, this situation, where a twodimensional surface is totally umbilic, is not generic and, in general, umbilics occur at isolated points in two dimensions and along lines in three dimensions. This is simply because $\Delta$ is two dimensional, and one requires both $\Delta_{1}=0$ and $\Delta_{2}=0$, meaning umbilics are codimension 2 . The presence of chirality $\left(q_{0} \neq 0\right)$ or spin-orbit coupling in the free energy Eq. (1) yields the generic situation.

Their dimensionality, coupled with the isotropic nature of $\nabla_{\perp} \mathbf{n}$ on umbilic lines, means that umbilics are associated with the centers of vortexlike distortions. For chiral free energies such Eq. (1), the preference for twist leads to umbilic profiles such as those shown in Figs. 1(a), 1(c), and 1(e), typically seen in materials with chiral interactions. For chiral vortices there is an immediate correspondence with "double twist" [8], traditionally written as $\partial_{i} n_{j}=-q_{0} \epsilon_{i j k} n_{k}$, which is equivalent to the condition of being on an umbilic line, with only twist deformations. Typical double twist configuration, such as shown in Fig. 1(e), are axisymmetric and contain an umbilic line along their centerline; however, this umbilic is not generic. In the generic case the umbilics are nonaxisymmetric, such as the two $\lambda$ lines in the dislocation shown in Fig. 1(a). They can still correspond to regions where the free energy is lower than that of the helical state, as long as the other aspects of the gradient tensor, splay and bend, are sufficiently small. Umbilic lines with local structures dominated by splay and bend distortions, Fig. 1(d), can also be energetically favorable for systems with a term of the form [81]

$$
\mathbf{E} \cdot((\mathbf{n} \cdot \boldsymbol{\nabla}) \mathbf{n}-\mathbf{n}(\boldsymbol{\nabla} \cdot \mathbf{n}))
$$

for an external field $\mathbf{E}$, replacing the chiral term in Eq. (1). This term corresponds to the flexoelectric effect in liquid crystalline systems [82] and to Rashba spin-orbit coupling in magnetic systems [81]. By an analogous argument to that given above, it is easy to see that umbilics with splay and bend distortions can correspond to local minima of a free energy with such a term. In this way the existence of Skyrmion lattices, such as those shown in Fig. 2, can be interpreted as due to the energetic preference for umbilic lines and the association between umbilic lines and Skyrmions, given by Eq. (20).

The decomposition Eq. (6) conveys the geometric character of the orientational order. In geometric terms the splay and twist correspond to the mean curvature and (a) ㅁㅁㅁㅁ000000000000 000000000000000000 000000000000000000 000000000000000000 घण000000000000000 ㅁㅁㅁ000000000000 ص日000001000000000 000000000100000000 000000000010000000

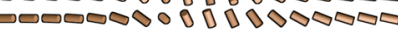
ㅂㅁㅁㅁ0000000000000 D00000000000000000 000000000000000000 00000000000000000

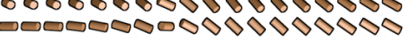

(c)

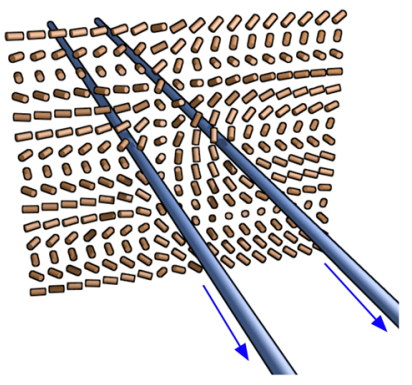

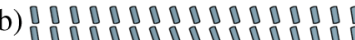

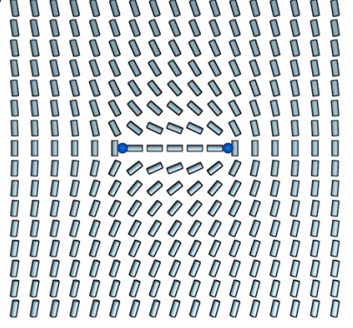

(d)

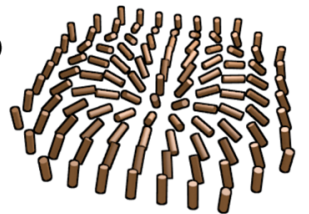

(e)

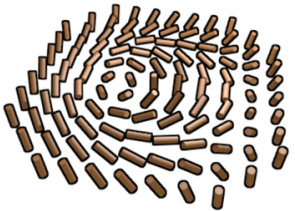

FIG. 1. Umbilic lines. (a) $\lambda^{+} \lambda^{-}$dislocation in a cholesteric. It contains two generic umbilics. This combination of two generic umbilics can be identified as half a Skyrmion, or a meron. (b) Eigenvector field of $\Pi$ for the dislocation. The two umbilics have opposite windings of the eigenvectors, but are counted with the same sign as $\mathbf{n}$ rotates by $\pi$ when passing from one to the other. (c) Umbilic lines can be oriented in three dimensions. (d), (e) Typical centers of vortices, based on curvature and torsion distortions, respectively, that correspond to winding number two nongeneric umbilics.

the mean torsion $[83,84]$ of the vector field, with the twist encoding the chirality of the order with surfaces of zero twist separating regions of different handedness. $\Delta$ can be thought of as a deviatoric, or spin-2, component of $\nabla \mathbf{n}$ that conveys the local "shear" of the vector field. Its eigenvectors can be used to define principal directions of the curvature of the local orientation. In the case where $\mathbf{n}$ is the normal to a surface, the transformation Eq. (6) is the derivative of the Gauss map, or shape operator of the surface, and the principal directions alluded to are the directions of principal curvature. In the vicinity of an umbilic we may write

$$
\Delta=|\Delta|\left[\begin{array}{cc}
\cos \theta & \sin \theta \\
\sin \theta & -\cos \theta
\end{array}\right],
$$

and around the umbilic the angle $\theta$ winds by an integer multiple of $2 \pi$. An umbilic is said to be generic if it has a winding number of \pm 1 . An example is shown in Fig. 1(a). Umbilics with higher winding do occur, and indeed those with winding +2 are often observed in vortices, double twist cylinders, and Skyrmions, with the degeneracy coming from an imposed axisymmetry, Figs. 1(d) and 1(e). This can persist in certain experimental settings, where there is either axisymmetry or hexagonal symmetry; 


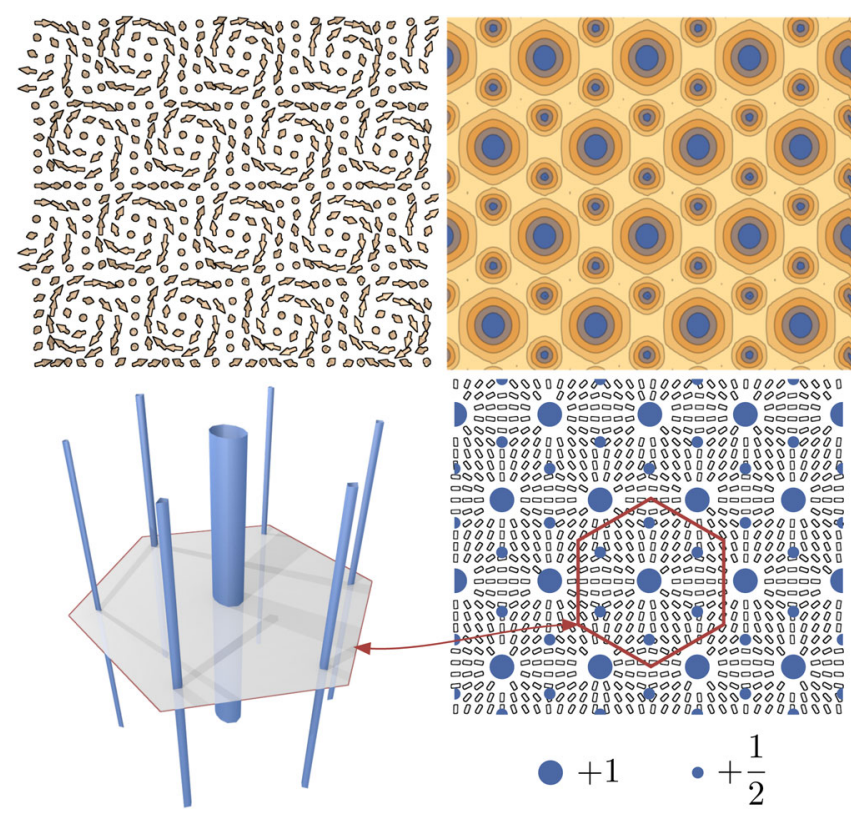

FIG. 2. Skyrmion lattice. Each unit cell possesses a single central $s=2$ umbilic, with the six outer $s=1$ umbilics each shared between three unit cells giving a total count of $2+6 \times 1 / 3=4$ umbilics $=1$ Skyrmion per unit cell. Top left: Unit vector field $\mathbf{n}$ in a Skyrmion lattice. Top right: Contour plot of $|\Delta|$ showing the umbilic lines forming a lattice of tubes. Bottom right: Eigenvector field of $\Pi$, illustrating the +1 winding around the central umbilics and $-1 / 2$ winding around the outer umbilics. Note that both are counted with the same sign as the orientation of $\mathbf{n}$ changes from one to another. Bottom left: Umbilics form lines in three dimensions.

however, from a structural point of view, they can be seen as a composite of winding \pm 1 umbilics, into which they break apart under perturbation. The generic umbilic points of a surface have been classified as stars, lemons, or monstars [64], according to the local profile that the principal curvature directions have in the vicinity of the umbilic point. The same local structure arises in the rotation of the eigenvectors of $\Delta$ around the umbilic lines of a generic vector field in three dimensions-it is not hard to see that the eigenvectors of $\Delta$ rotate by only half as much as $\Delta$ itself, i.e., by a half-integer multiple of $2 \pi$, this being the familiar phenomenon of a real Berry phase [70,71] — so that the local profile of an umbilic line may be classified in the same way as the umbilic points of a surface or the $C$ lines of generic electromagnetic fields [38].

We explain later that the windings just described are equivalent to the indices of the umbilics up to a sign, which arises because the orientation of $\mathbf{n}$ reverses from one umbilic to another. (The discrepancy is therefore absent if $\mathbf{n}$ is the normal to a surface, since then there is no reversal of its orientation between umbilics, and the index is simply the local winding of the eigenvectors of $\Delta$.) The sum of the indices of the umbilics yields a topological invariant, equal to 4 times the Skyrmion number of the texture on the two-dimensional surface. This calculation of the Skyrmion number is dual to the usual method of integrating a curvature form over a surface $\Sigma \subset \mathbb{R}^{3}$, which computes the degree of the map $\mathbf{n}: \Sigma \rightarrow S^{2}[37,85]$,

$$
q=\frac{1}{8 \pi} \int_{\Sigma} \epsilon_{a b c} n^{a} \partial_{i} n^{b} \partial_{j} n^{c} d x^{i} \wedge d x^{j}
$$

where all indices run from 1 through 3 . The duality can be viewed as entirely analogous to that in the classical differential geometry of surfaces between the GaussBonnet theorem-integral of the Gaussian curvatureand the calculation of the Euler characteristic using umbilic points of the surface. This correspondence allows one to localize the geometric distortions that characterize Skyrmions - the centers of the vortices-in a principled way.

This may be illustrated through the Skyrmion lattice of Fig. 2. Each hexagonal unit cell contains a single Skyrmion. When analyzed in terms of umbilics, one finds that the center of each hexagon corresponds to a nongeneric umbilic of winding number +2 , while every vertex marks the location of a generic umbilic with winding number -1 . The figure shows clearly that the eigenvectors of $\Delta$ rotate around the umbilics at half this rate with windings +1 and $-1 / 2$. This winding does not correspond to the index of the umbilic, however. In Fig. 2 the eigenvectors of $\Delta$ do not live in the viewing plane, but rather in the spatially varying plane of vectors with normal $\mathbf{n}$. On moving from a central umbilic to one with a $-1 / 2$ profile, $\mathbf{n}$ rotates by $\pi$, reversing the orientation of this plane. Accounting for this change in orientation assigns indices of the same sign to each umbilic in Fig. 2 ( +2 and +1 for each type, respectively). We show later that this can be phrased in terms of a natural orientation carried by each umbilic. Since each vertex is shared between three unit cells, it follows that the total count of umbilics per unit cell is $2+6 \times 1 / 3=4$, i.e., exactly 4 times the Skyrmion charge Eq. (10). As already remarked, this duality relation in determining the Skyrmion number between the integration of a curvature form and the count of umbilics is entirely analogous to the calculation of the Euler characteristic of a surface using either the GaussBonnet theorem or the umbilic points. The difference that arises here, illustrated by the example of the Skyrmion lattice, is that the count associated with the umbilics is not simply of their winding numbers, as in the case of surfaces, since $\mathbf{n}$ is not constrained to be a surface normal.

This correspondence also allows a robust identification of geometric distortions in materials that do not necessarily correspond to a traditional topological object. Such an example is given by the meron, or half-Skyrmion, which can be seen in magnetic films [45]. A meron can be thought of as carrying Skyrmion charge $\pm 1 / 2$ and as such does not correspond to a topologically well-defined object since Skyrmion charges are, by definition, integers. From the 
perspective of umbilic lines, however, a meron can be rigorously identified simply as a pair of generic umbilic lines or a single umbilic line of index \pm 2 . Figure 1(a) shows an image of a dislocation in helical order, which can be identified as a meron. Generic umbilics themselves correspond to even more elementary geometric distortions and in cholesterics correspond to the well-known $\lambda$ lines.

In two-dimensional systems umbilics are points, and give a correspondence between the geometric features of the order and topological distortions in the material. Moving up one spatial dimension, the situation becomes more complex and in three dimensions umbilic points become lines along which their surrounding vortices get extruded. Skyrmions themselves are more subtle in three dimensions, and can only be associated to an arbitrary measuring surface in the material, such as one enclosing a point defect, which must have a corresponding number of umbilic lines intersecting it. Umbilic lines therefore provide a complete, concise picture of the topological content of a three-dimensional texture, without resorting to integrals over local measuring surfaces. In a material domain with no boundaries, such as $\mathbb{R}^{3}$, umbilic lines can only end on singularities in $\mathbf{n}$. Indeed, as a consequence of Eq. (10), any point defect, or magnetic monopole, of charge $q$ must have umbilics of total winding $4 q$ emerging from it. This structure is illustrated in Fig. 3, where one sees the creation and annihilation of umbilic lines (and, consequently, a Skyrmion) by magnetic monopoles. Recently, this creation and annihilation has been experimentally observed [16] in chiral ferromagnets, and umbilic lines provide a precise physical localization of the lines of Skyrmion charge created by monopoles.

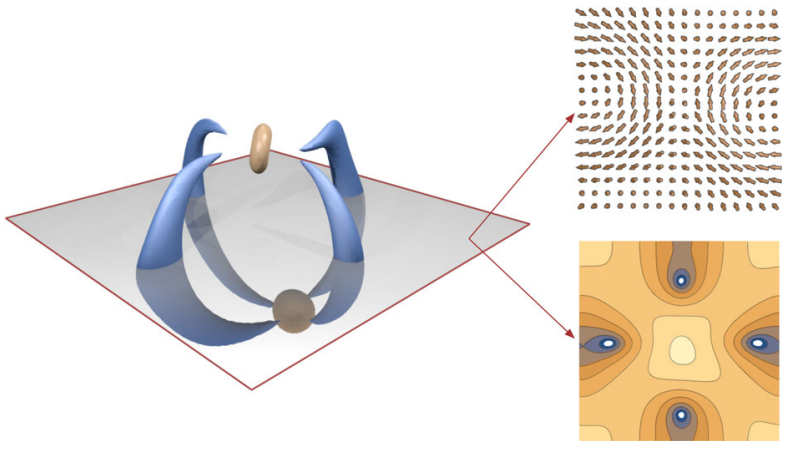

FIG. 3. Transient monopoles and umbilic lines observed in a simulated quench of the free energy [Eq. (1)]. Left: Contours of constant value of $|\Delta|$. The blue lines are umbilics, the beige spheres are monopoles (point defects). Each umbilic has winding $n=1$. They are all oriented similarly, so that the sum of the umbilics entering (leaving) the negative (positive) point defect is 4, in accordance with Eq. (20) and corresponding to the Skyrmion created between the monopoles. Top right: Cross section showing n on the shaded plane. As a map from the plane to $S^{2}$, it has degree 1, indicating the presence of a Skyrmion. Bottom right: Contour plot showing the magnitude of $\log (|\Delta|)$ on the shaded plane; the umbilics are clearly picked out.
Umbilic lines that do not end on defects or domain boundaries must instead form closed loops. These have been observed, though not identified as such, in several experiments, including torons [30] and nonsingular textures in colloidal dimers [35]; umbilics provide a rigorous way to characterize such objects, which have previously been described in an ad hoc fashion. In the same way that the local structure of orientational order leads to a global invariant of umbilics, namely Skyrmions, the local structure along umbilic lines leads to global topological invariants of umbilic loops, associated to their linking and twisting. These invariants have not yet been explored experimentally, but their association with helicity and Chern-Simons theory, discussed later, suggests deeper relevance to the physics of three-dimensional orientational order, making the study of closed umbilics of particular interest.

A canonical example of an umbilic loop is the toron, shown in Fig. 4, which contains a generic umbilic loop, with a monstar profile for the eigenvectors of $\Delta$, rather than the axisymmetric profile that was previously assumed. Indeed, with the methods we develop here, one can show that the symmetries of both the toron and the colloidal dimer texture necessitate the creation of this umbilic loop. Both these axisymmetric systems consist of two point
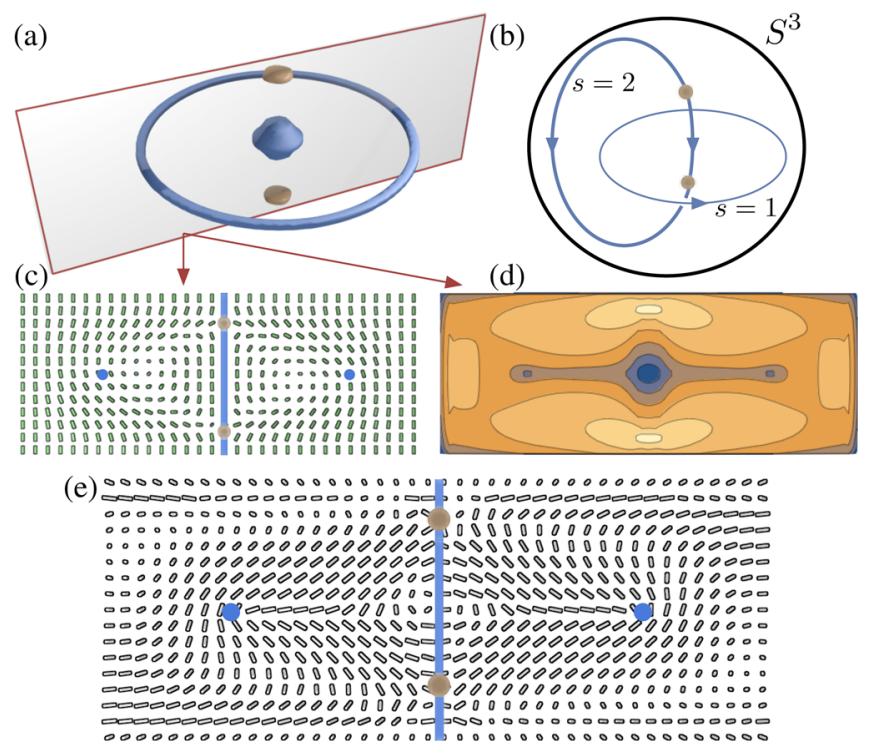

FIG. 4. Umbilic lines in a toron. (a) Simulation results showing point defects and characteristic umbilic loop in a toron. The umbilic loop is transverse and has zero self-linking number. The point defects have opposite charge; due to lattice effects the simulation does not resolve the umbilic lines connecting them, though they are clear in the topology of the eigenvector field. (b) Diagram illustrating the umbilic structure of the toron in $S^{3}$ when the boundary of the cell is collapsed to a point. Note that the umbilic loops connecting the point defects each have strength $s=2$, for a total of 4, as required by Eq. (20). (c) Simulation results showing director field for the toron. (d) Contour plot showing the value of $|\Delta|$. (e) Eigenvector field of $\Pi$. Note that the umbilic loop is generic and has a monstar profile. 
defects (in the colloidal dimer textures these are effective point defects created by colloidal particles), with an umbilic loop lying in the plane equidistant from the two defects. The existence of this umbilic loop requires that the two point defects have the same local structure, but opposite signs - either both hyperbolic in the case of the toron or both radial in the case of the colloidal dimer texture-and suggests that the creation of such monopole pairs in other systems may well lead to the formation of umbilic loops.

As mentioned above, the global invariants of umbilic loops come about through their twisting and linking. If an umbilic forms a closed loop, then the winding of the angle $\theta$, given in Eq. (9), along the contour length of the umbilic (a longitude) conveys an integer invariant that depends on a choice of longitude and of a basis, $\left\{\mathbf{d}_{1}, \mathbf{d}_{2}, \mathbf{n}\right\}$, along the loop. This integer is related to a self-linking number of the umbilic loop, which is itself related to the Hopf invariant and structures from contact topology. This connection, between linking of umbilics and the Hopf invariant of $\mathbf{n}$, can be illustrated in Fig. 9, which can be viewed as the linking of two nongeneric umbilics.

An additional aspect of the local geometry is worth emphasizing. The complex structure $J=\left[\begin{array}{cc}0 & -1 \\ 1 & 0\end{array}\right]$ acts on the shape operator Eq. (6) by simple composition to produce an equivalent linear transformation:

$$
\begin{aligned}
\chi & =J \circ \nabla_{\perp} \mathbf{n} \\
& =-\frac{\mathbf{n} \cdot \boldsymbol{\nabla} \times \mathbf{n}}{2}\left[\begin{array}{ll}
1 & 0 \\
0 & 1
\end{array}\right]+\frac{\boldsymbol{\nabla} \cdot \mathbf{n}}{2}\left[\begin{array}{cc}
0 & -1 \\
1 & 0
\end{array}\right]+\left[\begin{array}{cc}
-\Delta_{2} & \Delta_{1} \\
\Delta_{1} & \Delta_{2}
\end{array}\right] .
\end{aligned}
$$

This transformation, although equivalent to the shape operator, is independent from it in the sense that they are orthogonal with respect to the natural inner product between matrices, $\operatorname{Tr}\left(\chi^{T} \nabla_{\perp} \mathbf{n}\right)=0$, a property that turns out to be of some importance in the analysis of the topology of umbilics. $\chi$ is the chirality pseudotensor introduced recently by Efrati and Irvine [78] in their study of chiral materials; our description of it provides a complementary perspective to theirs. The spin-2 component $\Pi=J \circ \Delta$ carries equivalent information to $\Delta$. Its eigenvectors can be thought of as defining directions of principal torsion (or twist) and are simply rotated relative to those of $\Delta$ by $\pi / 4$ about the director. Nonetheless, for materials with chiral interactions or structure, it is these directions, rather than the eigenvectors of $\Delta$, that are often more visibly recognizable, as is elegantly illustrated in the experiments of Armon et al. [86] on the opening of chiral seed pods, and those of Efrati and Irvine [78] on stretched elastic sheets. For this reason, we plot the eigenvectors of $\Pi$ rather than those of $\Delta$ in all figures in this paper. The eigenvectors of $\chi$ have been used recently [36] to give a definition of the pitch axis for a cholesteric. In the case where $\boldsymbol{\nabla} \cdot \mathbf{n}=0$, the eigenvectors of $\Pi$ coincide with those of $\chi$, and the principal directions of torsion can be identified with the pitch axis from $\chi$. In general, however, this is not the case. Indeed, the (real) eigenvectors of $\chi$ do not always exist and, as such, cannot provide global topological information in a manner analogous to that provided by $\Delta$ (or $\Pi$ ).

\section{UMBILICS IN LINE FIELDS AND THE BLUE PHASES}

In liquid crystalline phases the orientational order is linelike rather than vectorial. Perhaps the most noticeable feature of this is the presence of line defects, called disclinations, in liquid crystals $[87,88]$, which make manifest the nonorientability and are absent in materials with vector order. However, they can arise even in vectorordered materials if the vector couples to an internal degree of freedom to give nematiclike symmetry; examples include thin films of ${ }^{3} \mathrm{He}$ [89] and spin-1 polar condensates [90]. As the director is nonsingular along umbilics, the effect of the nonorientability is primarily restricted to their global identification and structure. Indeed, since umbilics can be identified from the local structure of the director gradients, they have the same local description for line fields as they do for vector fields. One need only choose an orientation for the line field, which may always be done locally, i.e., in regions that do not contain disclination lines.

Rather than present a detailed account of the analysis of line fields, we focus on simple illustrations and a practical means for identifying umbilics globally, using one particular physical system - the cholesteric blue phases. These remarkable materials are characterized by periodic orientational order corresponding to crystalline space groups, while remaining three-dimensional fluids. Two distinct cubic structures, BPI with space group $\mathrm{O}^{8-}\left(I 4_{1} 32\right)$ and BPII with space group $\mathrm{O}^{2}\left(\mathrm{P}_{2} 32\right)$, are observed on varying temperature or the amount of chiral dopant $[8,9,91]$, while several other structures can be induced by applied electric field effects [92-94] or confinement [95], and there is also an amorphous phase, known as BPIII [96]. Their structure arises because the local optimal configuration for the chiral free energy is one of double twist (illustrated in Figs. 1 and 6), but this is only locally favorable and the structure becomes energetically unstable if it extends too far. This leads to a frustrated arrangement where locally preferred regions of double twist form periodic arrays interwoven by a network of disclination lines [8,9]. The blue phases have a long-standing association with Skyrmions in chiral magnets, with which they may be considered analogous. This analogy continues to stimulate considerable interchange of ideas between the two fields $[3,29,97-99]$.

It is usual to describe the order in liquid crystals, especially in numerical simulations, using a tensor order parameter, called the $Q$ tensor, that captures the nonorientability of the director field [75]. The $Q$ tensor is proportional to the deviatoric part of the dielectric tensor or 
the second moment of the molecular orientational distribution function. The director field can be recovered from it as the eigenvector associated to the largest eigenvalue. In typical nematic materials, the $Q$ tensor is uniaxial and we have $\mathbf{Q} \propto \mathbf{n} \otimes \mathbf{n}-\frac{1}{3} \square$. Our first objective is to describe how umbilics may be identified from the $Q$ tensor. The shape operator Eq. (6), and its deviatoric part $\Delta$, change sign under $\mathbf{n} \rightarrow-\mathbf{n}$, and so do not directly give rise to objects compatible with nematic symmetry. However, interestingly, the chirality pseudotensor Eq. (11), and its deviatoric part $\Pi$ Eq. (17), continue to be globally well defined in the same form even when the director is nonorientable, so that $\Pi$ admits an immediate extension to a " $Q$-tensor version." We use this to identify umbilics in line fields.

The linear transformation $\Pi$, defined fully in Eq. (17), can be embedded in a $3 \times 3$ matrix and expressed, in a Cartesian basis, as

$$
\begin{aligned}
\Pi_{i j}= & \frac{1}{4} \epsilon_{i l k}\left[n_{l} \partial_{k} n_{j}+n_{l} \partial_{j} n_{k}-n_{j} n_{l} n_{m} \partial_{m} n_{k}\right] \\
& +\frac{1}{4} \epsilon_{j l k}\left[n_{l} \partial_{k} n_{i}+n_{l} \partial_{i} n_{k}-n_{i} n_{l} n_{m} \partial_{m} n_{k}\right] .
\end{aligned}
$$

The issue at hand is what a suitable replacement should be if we express the orientational order using a $Q$ tensor rather than the director field. The usual approach to obtaining such a replacement is to adopt algebraic methods rather than geometric ones [100]; one constructs a list of potential expressions and selects on the basis of how they reduce when the $Q$ tensor is written in terms of the director field. Following this procedure, we find that the expression

$$
\begin{aligned}
\tilde{\Pi}_{i j}= & \frac{1}{4} \epsilon_{i l k}\left[2 Q_{l m} \partial_{k} Q_{j m}+Q_{j m} \partial_{k} Q_{l m}\right. \\
& \left.+Q_{l m} \partial_{j} Q_{k m}-Q_{j l} \partial_{m} Q_{k m}\right] \\
& +\frac{1}{4} \epsilon_{j l k}\left[2 Q_{l m} \partial_{k} Q_{i m}+Q_{i m} \partial_{k} Q_{l m}\right. \\
& \left.+Q_{l m} \partial_{i} Q_{k m}-Q_{i l} \partial_{m} Q_{k m}\right]
\end{aligned}
$$

reduces to $\Pi_{i j}$ when the $Q$ tensor is uniaxial, with constant magnitude, $Q_{i j} \propto n_{i} n_{j}-\frac{1}{3} \delta_{i j}$. We subtract its trace and adopt it as an appropriate $Q$-tensor analog of $\Pi$. In simulations, the umbilic lines can then be identified using isosurfaces where the norm of $\tilde{\Pi}$ drops below a threshold value.

Illustrations are given in Fig. 5 for some standard blue phase textures. The umbilics coincide with structural motifs that have been identified previously, either through symmetry considerations [101] or by taking a singular value decomposition of the $Q$ tensor [80]. These motifs are the axes of double twist cylinders [8,9]. Two remarks are in order concerning this. First, the pattern of umbilic lines in BPII (see Fig. 5) does not correspond to the array of double
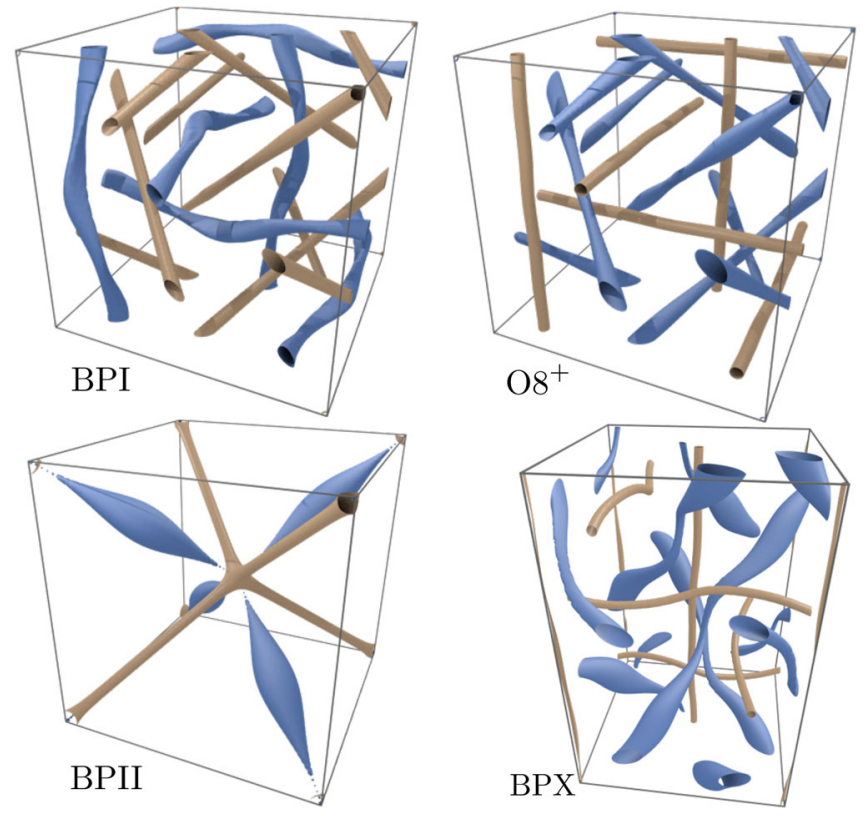

FIG. 5. Umbilic lines in the blue phases. The umbilics are indicated by blue isosurfaces, while the network of disclination lines is shown by milk chocolate isosurfaces. The textures BPI and BPII are the cubic structures observed experimentally. $\mathrm{O}^{+}$is a cubic structure with the same space group as BPI but an interchange between disclination lines and umbilics. BPX is a tetragonal texture observed in an applied electric field. The arrangement of disclinations and umbilics is the same as in $\mathrm{O}^{8+}$, although the symmetry is different.

twist cylinders traditionally depicted in the literature. In the traditional depiction the double twist cylinders are taken along the twofold axes lying in the faces of the conventional unit cell and, indeed, inspection by eye reveals these lines to have the same general structure as model double twist cylinders [8] (see Fig. 1). However, in a $Q$-tensor description of BPII these are not degeneracies where the local structure is axisymmetric, as in the standard idealized picture of a double twist cylinder; rather, they are twofold lines where the $Q$ tensor is maximally biaxial $[80,102,103]$. Second, it is perhaps worth emphasizing that the umbilics identified in all other blue phase textures also differ from the standard idealized picture of a double twist cylinder. This is because the idealized axisymmetry of the latter constrains it to be a degenerate winding +2 umbilic, whereas those that are found in periodic textures are generic umbilics, typically with a $-1 / 2$ profile for the eigenvectors of $\tilde{\Pi}$, i.e., a star umbilic.

We have given here only cursory illustrations for periodic blue phases with crystalline space groups. It would be of interest to also study the structure of the amorphous BPIII in terms of umbilics, as well as their behavior in transitions and rheology. The $Q$-tensor version of the linear transformation $\Pi$ that we introduce here is motivated on algebraic grounds rather than the geometrical 
considerations that the rest of our work is founded upon. It is clear that geometrical considerations can be developed for traceless symmetric tensors, parallel to our description of orientational order. It would be interesting to do so and compare the outcome with the algebraic construction we have given.

In the remainder of this paper we return to the setting of vector orientational order and focus on the global topological aspects represented by umbilic lines. However, before doing so we summarize here how those considerations extend to nonorientable line fields. Orientational order defines a splitting of the tangent bundle $T \mathbb{R}^{3} \cong$ $L_{\mathbf{n}} \oplus \xi$ whether it is orientable or not, although the line bundle $L_{\mathbf{n}}$ is nontrivial in the nonorientable case, with the consequence that the director $\mathbf{n}$ is not globally defined (as a vector). Rather, the projector $\mathbf{n} \otimes \mathbf{n}$ provides a section of $L_{\mathbf{n}} \otimes L_{\mathbf{n}}$. Similarly, the shape operator has to be modified so as to be presented as a section of the twisted bundle $\xi^{*} \otimes \xi \otimes L_{\mathbf{n}}$ [104]. The same description holds for the global character of umbilics in that they still furnish a representative of the Poincare dual to twice the Euler class of the orthogonal 2-plane bundle $\xi$. Interestingly, for line fields with knotted disclination lines, the Euler class of the orthogonal 2-plane bundle is usually torsion [52], which the usual Chern-Weil theory of curvature forms is insensitive to. (The curvature form represents only the integral part of the cohomology $[105,106]$.) The integral in Eq. (23) thus does not provide any information in such cases. However, as the umbilic lines are Poincaré dual to twice the Euler class of $\xi$ (and $4[\mathbf{n}]$ ), they continue to capture the topology of the orientational order, even when it is torsion, modulo elements of order 4. As discussed earlier, and shown in Fig. 1, one can identify umbilics with $\lambda$ lines in cholesterics. In this way, Eq. (20) can be seen as a global constraint on the $\lambda$ lines in a cholesteric, as determined by the topology of $\mathbf{n}$, with additional subtlety associated with elements in $H^{2}(M)$ of order 2 and 4 . We anticipate that the elements of order 4 are associated with $\tau$ lines. For the elements of order 2 the orthogonal 2-plane bundle $\xi$ admits a global section and we anticipate that these are associated with distinct "cholesteric ground states" [104].

\section{GLOBAL DEFINITION OF UMBILICS AND THE TOPOLOGY OF VECTOR FIELDS}

The umbilic lines we identify convey topological information about the vector field $\mathbf{n}$, specifically, the Euler class of the orthogonal 2-plane bundle $\xi$, through a combination of the Gauss-Bonnet-Chern theorem [107-110] and Poincare duality. The curvature of a vector bundle represents a characteristic cohomology class that can be integrated over homology cycles to produce topological invariants (Euler numbers). By Poincaré duality this can be viewed as a homology class and the umbilics furnish a representative of this Poincaré dual. We develop this general topology of orientational order in terms of a natural connection on the vector bundle that the spin-2 transformation $\Delta$ takes values in.

As before, let $\mathbf{n}$ be a unit vector field in $\mathbb{R}^{3}$; then, it defines a splitting of the tangent bundle

$$
T \mathbb{R}^{3} \cong L_{\mathbf{n}} \oplus \xi
$$

into a real line bundle $L_{\mathbf{n}}$ of vectors parallel to $\mathbf{n}$ and a rank2 real vector bundle $\xi$ of vectors orthogonal to $\mathbf{n}$. Examples of this splitting are shown in Fig. 6. The gradients of $\mathbf{n}$ take values in $T \mathbb{R}^{3} \otimes \xi \cong\left(L_{\mathbf{n}}^{*} \otimes \xi\right) \oplus\left(\xi^{*} \otimes \xi\right)$, with the first part containing the bend deformations and the second the perpendicular gradients given locally in Eq. (6). By complete analogy with the classical differential geometry of surfaces, we call these perpendicular gradients the shape operator of the orientational order and think of it as a linear transformation on the orthogonal 2-planes.

The shape operator decomposes in the following way. Local rotations about the director field correspond to a local symmetry and endow the bundle $\xi$ with a natural $\mathrm{SO}(2)$ action, under which the tensor product $\xi^{*} \otimes \xi$ splits into a direct sum $\mathcal{I} \oplus \mathcal{J} \oplus E$, where $\mathcal{I}$ and $\mathcal{J}$ are trivial line bundles and $E$ is a rank-2 vector bundle. Correspondingly, the shape operator has the decomposition

$$
\nabla_{\perp} \mathbf{n}=\frac{\boldsymbol{\nabla} \cdot \mathbf{n}}{2} \rrbracket_{\xi}+\frac{\mathbf{n} \cdot \boldsymbol{\nabla} \times \mathbf{n}}{2} J+\Delta,
$$

given previously in Eq. (6). Here, $\mathbb{\square}_{\xi}$ is the identity transformation and $J=\mathbf{n} \times$ is a complex structure on $\xi$, now defined globally, while $\Delta$ has the global definition

$$
\Delta(\mathbf{v})=\frac{1}{2}[(\mathbf{v} \cdot \boldsymbol{\nabla}) \mathbf{n}+\mathbf{n} \times(\mathbf{n} \times \mathbf{v} \cdot \boldsymbol{\nabla}) \mathbf{n}],
$$

for any $\mathbf{v}$ in $\xi$. The umbilics are identified with the zeros of $\Delta$; they encode the topology of the orientational order.

Without repeating too much of the local description given previously, we record simply that composition with the complex structure again yields the chirality pseudotensor [78], $\chi=J \circ \nabla_{\perp} \mathbf{n}$, and that the spin-2 component of this, $\Pi=J \circ \Delta$, can be expressed globally as
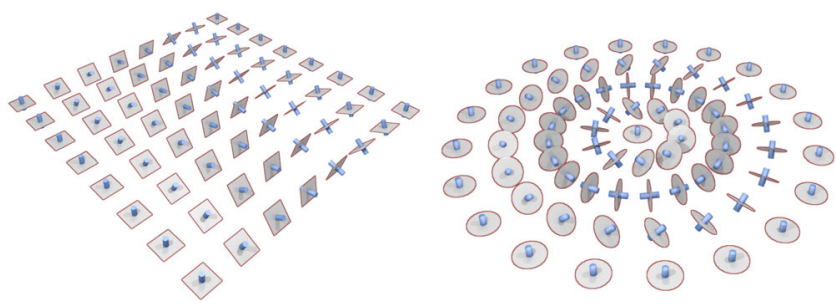

FIG. 6. The splitting $T \mathbb{R}^{3} \cong L_{\mathbf{n}} \oplus \xi$ induced by orientational order. Left: A simple helical director profile, corresponding to the ground state of a cholesteric liquid crystal or helimagnet. Right: An idealized, axisymmetric Skyrmion profile, the central portion of which is a standard idealized model of double twist cylinders in blue phases. 


$$
\Pi(\mathbf{v})=\frac{1}{2}[\mathbf{n} \times(\mathbf{v} \cdot \boldsymbol{\nabla}) \mathbf{n}-(\mathbf{n} \times \mathbf{v}) \cdot \boldsymbol{\nabla n}],
$$

for any $\mathbf{v}$ in $\xi$, and is also a section of $E$. We reiterate that although $\Pi$ and $\Delta$ may be obtained one from the other by composition with the complex structure, they are orthogonal with respect to the natural inner product $\langle\Pi, \Delta\rangle=\frac{1}{2} \operatorname{Tr}\left(\Pi^{T} \Delta\right)$. At the same time, they share the same magnitude and the same zero set, the umbilics $\mathcal{U}$. Thus, away from umbilic lines, $\Pi$ and $\Delta$ represent two nonzero orthogonal sections of $E$, and so they provide a basis for $\left.E\right|_{\mathbb{R}^{3}-\mathcal{U}}$ and also define a natural connection 1-form:

$$
A=\frac{\langle\Pi, \nabla \Delta\rangle}{\langle\Delta, \Delta\rangle}
$$

This 1-form conveys the fundamental topology of the orientational order.

We give in Eq. (18) a global definition of $A$ that emphasizes its naturality and makes clear how it arises. In a local chart it can be represented in terms of the eigenvectors, $\mathbf{p}^{+}$and $\mathbf{p}^{-}$, of $\Delta$ and has components $A_{i}=2 p_{a}^{-} \partial_{i} p_{a}^{+}$, an expression perhaps closer in form to the usual way of writing Berry connections. However, one must keep in mind that this representation is only local in the present context; otherwise, it only serves to obfuscate the natural structure. The exterior derivative of $A$, $\Omega_{E}=d A$, is the (Berry) curvature of the vector bundle $E$. It is equal to twice the curvature of $\xi$ on account of $E$ being a spin-2 subbundle of $\xi^{*} \otimes \xi$. (This is also evident from the local expression in terms of the eigenvectors $\mathbf{p}^{ \pm}$.)

A fundamental property of an umbilic is given by the integral of the connection 1-form [Eq. (18)] around any simple closed loop $\gamma$ encircling the umbilic. This is the holonomy of the connection, or Berry phase. Let $\Sigma$ be an oriented surface with boundary $\gamma$, as in Fig. 7. If $\Sigma$ is generic, then the umbilics will intersect it transversally in some number of distinct points. Let $\left\{D_{i}\right\}$ be disjoint small disks in $\Sigma$ about each of these intersection points and denote their boundaries by $\left\{C_{i}\right\}$. Then $|\Delta|$ is nowhere zero on $\Sigma-\left\{D_{i}\right\}$ and $\partial\left(\Sigma-\left\{D_{i}\right\}\right)=\gamma \sqcup\left\{-C_{i}\right\}$, so that by Stokes's theorem

$$
\int_{\gamma} A-\sum_{i} \int_{C_{i}} A=\int_{\Sigma-\left\{D_{i}\right\}} d A=\int_{\Sigma-\left\{D_{i}\right\}} \Omega_{E}
$$

To extend the analysis over the umbilics, we can introduce a local trivialization of $L_{\mathbf{n}} \oplus \xi$ on each of the $D_{i}$, which we denote by the orthonormal basis $\left\{\mathbf{d}_{1}, \mathbf{d}_{2}, \mathbf{n}\right\}$. Then $\mathbf{e}_{1}=$ $\mathbf{d}_{1} \otimes \mathbf{d}_{1}-\mathbf{d}_{2} \otimes \mathbf{d}_{2}$ and $\mathbf{e}_{2}=\mathbf{d}_{1} \otimes \mathbf{d}_{2}+\mathbf{d}_{2} \otimes \mathbf{d}_{1}$ form a local basis for $E$. In such a basis, $\Delta=|\Delta| \cos \theta \mathbf{e}_{1}+|\Delta| \sin \theta \mathbf{e}_{2}$ and $A=d \theta+2 \omega$, where $\omega_{i}=\left(\mathbf{d}_{2}\right)_{a} \partial_{i}\left(\mathbf{d}_{1}\right)_{a}$ is the connection 1 -form on $\xi$ for this basis (the factor of 2 accounts

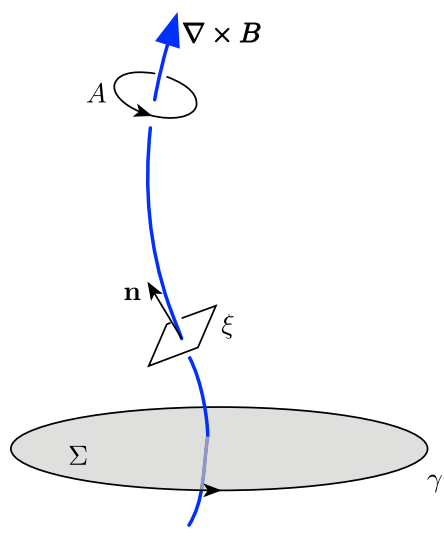

FIG. 7. Anatomy of an umbilic line. Top: The vector $\boldsymbol{\nabla} \times \boldsymbol{B}$ is tangent to generic umbilic lines, giving them a natural orientation. This orientation is compatible with that induced by $A$. Middle: The vector field $\mathbf{n}$ and associated orthogonal plane $\xi$. Note that, in general, the tangent vector of the umbilic does not align with $\mathbf{n} . \xi$ is oriented by $\mathbf{n}$ and so gives a natural cycle along which to measure the winding. For a generic umbilic, if $\mathbf{n} \cdot \boldsymbol{\nabla} \times \boldsymbol{B}>0$, then the eigenvectors of $\Delta$ have a $+1 / 2$ profile, and if $\mathbf{n} \cdot \boldsymbol{\nabla} \times \boldsymbol{B}<0$, the eigenvectors have a $-1 / 2$ profile. Bottom: A measuring cycle $\gamma$ bounding a surface $\Sigma$. Whether $\gamma$ measures the winding of the umbilic as positive or negative depends on whether the umbilic — oriented by $\boldsymbol{\nabla} \times \boldsymbol{B}$-intersects $\Sigma$ positively or negatively.

for $E$ being a spin-2 bundle). With this, one finds at once the Gauss-Bonnet-Chern theorem in the form

$$
\begin{aligned}
\frac{-1}{2 \pi} \int_{\Sigma} \Omega_{E}+\frac{1}{2 \pi} \int_{\gamma} A & =\sum_{i} \frac{1}{2 \pi} \int_{C_{i}} d \theta \\
& =\sum_{i} \operatorname{index}_{\Sigma} U_{i},
\end{aligned}
$$

which defines the index of an umbilic, an integer associated with its intersection with a surface. The sum of these over all of the umbilics is an Euler number of the bundle $E$. Note that, as alluded to earlier, the index of an umbilic is not simply the winding number of the angle $\theta$ that characterizes the local profile, as that is measured relative to a local basis for $\xi$, while the index uses the orientation on the surface $\Sigma$; there is, therefore, an additional sign according to whether $d \theta$ is cooriented with $C_{i}$ or not.

It is instructive to contrast this situation, where the type of profile does not carry topological information, with that of umbilical points of surfaces where the type of profile does carry topological information about the sign of the winding. This occurs because in the case of surfaces $\mathbf{n}$ is fixed to be the normal to $\Sigma$, and so $d \theta$ is always cooriented with $C_{i}$.

One can understand this relation between the profile and index in terms of oriented umbilics. The 1-form $A$ circulates around umbilic lines, orienting them such that the circulation is counterclockwise. If one orients umbilics 
in this way, the expression for the index in Eq. (20) is rewritten as

$$
\operatorname{index}_{\Sigma} U_{i}=s_{i} \operatorname{Int}\left(U_{i}, \Sigma\right),
$$

where $\operatorname{Int}\left(U_{i}, \Sigma\right)$ is the signed number of intersections of $U_{i}$ with $\Sigma$ and $s_{i} \in \mathbb{N}$ is the absolute strength of the umbilic $U_{i}$. Examples illustrating this are shown in Figs. 1 and 2; we describe only the latter (the former is essentially identical). Figure 2 shows a Skyrmion lattice, a well-known structure seen in a variety of systems [15,17-19,28,61,111-113]. The umbilics form a lattice, with $s=2$ umbilics each surrounded by six $s=1$ umbilics. The eigenvectors of $\Pi$ show the central umbilic having a +1 profile, and the six outer umbilics a $-1 / 2$ profile. All the umbilics have the same orientation induced by $A$, and so their intersection numbers, and thus indices in Eq. (20), are all positive. For the +1 profile, $-1 / 2$ profile, and the orientations to all be consistent, it is necessary that $\mathbf{n}$ rotates by $\pi$ when passing from the central umbilic to an outer one.

The curvature of the bundle $\xi$ can be expressed in terms of the director field as

$$
\Omega_{\xi}=-\frac{1}{2} \epsilon_{a b c} n^{a} \partial_{i} n^{b} \partial_{j} n^{c} d x^{i} \wedge d x^{j} .
$$

This is because each 2-plane of the bundle $\xi$ is explicitly embedded in $\mathbb{R}^{3}$ (see Fig. 6) and the expression is just the extrinsic definition of curvature in terms of the (generalized) Gauss map. Equally, it follows from an explicit calculation in local coordinates for any particular texture, say, a radial hedgehog. A third way to establish it is through a calculation in the style of the Mermin-Ho relation [10], a good account of which has been given by Kamien [114]. The curvature of $E$ is equal to twice this, $\Omega_{E}=2 \Omega_{\xi}$, since $E$ is a spin-2 subbundle of $\xi^{*} \otimes \xi$. Therefore, when $\Sigma$ is closed, or the boundary term vanishes, we have

$$
\begin{aligned}
\sum_{i} s_{i} \operatorname{Int}\left(U_{i}, \Sigma\right) & =\frac{1}{2 \pi} \int_{\Sigma} \epsilon_{a b c} n^{a} \partial_{i} n^{b} \partial_{j} n^{c} d x^{i} \wedge d x^{j} \\
& =4 q,
\end{aligned}
$$

or the sum of the indices of the umbilics piercing $\Sigma$ is equal to 4 times the Skyrmion number. This establishes a general relationship between Skyrmions and umbilics. This is illustrated in Fig. 2, where each unit cell contains one $s=2$ umbilic and six $s=1$ umbilics, each of which is shared between three unit cells, giving a total count of $2+6 \times 1 / 3=4$; i.e., there is one Skyrmion in each unit cell. This approach can also be extended to the characterization of merons, or half-Skyrmions, which can be thought of as a single $s=2$ umbilic, or two $s=1$ umbilics, as in the case of the cholesteric dislocation, shown in Fig. 1.
This correspondence between Skyrmions and umbilics can be seen as an instance of Poincaré duality, giving a natural way to localize Skyrmions to a set of points or lines. The set of umbilics $\mathcal{U}$ in a three-dimensional system $M$ gives a representative of a cycle in $H_{1}(M)$. The Poincaré dual of this is the cocycle $e(E) \in H^{2}(M)$, the Euler class, which depends only on the topology of the bundle $E$ and, hence, $\mathbf{n}$, and gives a global constraint on the total number of umbilic lines in the system in terms of the topology of $\mathbf{n}$. As a spin-2 vector bundle, $e(E)=2 e(\xi)=4[\mathbf{n}]$, where $[\mathbf{n}] \in H^{2}(M)$ is the cocycle represented by $\mathbf{n}$ [115]. In the absence of torsion in $H^{2}(M)$ [116], there is a canonical representative of the cocycle $4[\mathbf{n}]$ as a 2 -form, which is given through the Chern-Weil homomorphism by $\operatorname{Pf}\left(-\Omega_{E} / 2 \pi\right)$, which leads to Eq. (20).

An example of the Euler class as a global constraint on umbilics is given by the case of monopoles, illustrated in Fig. 3. These are point defects in the magnetization that mediate changes in the number of Skyrmions [16]. In the local neighborhood of a point defect the topology of $\mathbf{n}$ is described by an integer $q \in \pi_{2}\left(S^{2}\right) \cong \mathbb{Z}$, the charge of the point defect. Through Eq. (23) one finds that a sphere surrounding this point defect must be pierced by umbilics of total index 4q. In Fig. 3 we show a simulation of two \pm 1 point defects, or monopoles, in a chiral ferromagnet. There are four umbilics, each of generic type, ending on the point defects in a manner reminiscent of Dirac strings [107]. On a slice in the midplane of the cell $\mathbf{n}$ contains a Skyrmion, illustrating the well-known creation and annihilation of Skyrmions in terms of point defects, observed experimentally in Ref. [16].

\section{LOCAL PROFILES OF UMBILIC LINES}

Simple examples of umbilic lines, such as the Skyrmion lattice in Fig. 2, possess translational symmetry along the umbilic, with the tangent vector of the umbilic pointing parallel to $\mathbf{n}$. Both these situations are not generic; the local profile of umbilic lines may change along their length and the tangent vector of the line does not have to be parallel to n. To describe how these quantities can vary, one needs to understand the local structure of umbilic lines, the focus of this section. Ultimately, this local description will lead to the definition of global linking invariants or umbilic loops in the next section. This can be considered analogous to the situation earlier, where the local structure of umbilics in orientational order led to global invariants, namely, Skyrmions.

Generic umbilic lines carry a canonical orientation through the vector $B$, defined as

$$
B^{i}=\left\langle\Pi, \partial^{i} \Delta\right\rangle=\langle\Delta, \Delta\rangle \delta^{i j} A_{j},
$$

which assigns a tangent vector to the umbilic, provided it is generic, as follows. If, in a local trivialization, 
$\Delta=s_{1} \mathbf{e}_{1}+s_{2} \mathbf{e}_{2}$, then an umbilic is defined as the intersection of the two surfaces $s_{1}=0$ and $s_{2}=0$, and so its tangent vector will be $\boldsymbol{\nabla} s_{1} \times \nabla s_{2}$. Note that while the values of $s_{1}$ and $s_{2}$ depend on the choice of basis, the tangent vector does not. In this local form we can write

$$
B=s_{1} \nabla s_{2}-s_{2} \nabla s_{1}+2\left(s_{1}^{2}+s_{2}^{2}\right) \omega,
$$

so that on an umbilic we have $\boldsymbol{\nabla} \times \boldsymbol{B}=2 \boldsymbol{\nabla} s_{1} \times \boldsymbol{\nabla} s_{2}$, and, hence, the vector $\boldsymbol{\nabla} \times \boldsymbol{B}$ points along umbilic lines, canonically orienting them. As indicated in Fig. 7, this orientation is compatible with that induced by the circulation in $A$ previously discussed. As alluded to in Figs. 1 and 2, the discrepancy between the type of profile and index of an umbilic depends on the relative orientation between $\mathbf{n}$ and the umbilic line. To measure whether the eigenvectors of a generic umbilic have winding $\pm 1 / 2$, one should integrate $A$ along a contour $\gamma$ chosen such that its orientation matches the orientation imparted to $\xi$ by $\mathbf{n}$. One can then show that the local profile is $\pm 1 / 2$ depending on

$$
\operatorname{sgn}(\mathbf{n} \cdot \boldsymbol{\nabla} \times \boldsymbol{B}),
$$

the analysis being identical to that given by Berry for $C$ lines in electromagnetic fields [117]. The freedom between the relative orientations of umbilic lines and $\mathbf{n}$ means that $\mathbf{n} \cdot \boldsymbol{\nabla} \times \boldsymbol{B}$ can pass continuously through zero, changing the type of profile from $\pm 1 / 2$ to $\mp 1 / 2$, in a similar manner to nematic disclinations [87].

This quantity $\mathbf{n} \cdot \boldsymbol{\nabla} \times \boldsymbol{B}$ can be used to further characterize the local structure of umbilic lines. Along an umbilic $\Delta$ vanishes and generically we can expect it to vanish linearly. Its local profile is then given by the form of the linear order terms in $\Delta$ in the immediate vicinity of the umbilic. Equivalently, generically we can expect the gradients of $\Delta$ to be nonzero on the umbilic, and the local profile is then given by the structure of $\nabla \Delta$ evaluated on the umbilic. As usual, we split the gradients into the components parallel to $\mathbf{n}$ and those perpendicular to it, and consider only the latter. The nonzero part of these transverse gradients, evaluated on the umbilics, takes values in $\xi^{*} \otimes E$, so it is this part that we need to focus on. A section of this bundle characterizing the local profile of the umbilic can be constructed from $\nabla \Delta$ as follows. If $\mathbb{\square}_{E}$ is the projection onto $E$ in $T \mathbb{R}^{3} \otimes T \mathbb{R}^{3}$, then we define the differential operator $\nabla^{\xi}$ as $\boldsymbol{\nabla}^{\xi} \Delta=\rrbracket_{\xi} \boldsymbol{\nabla} \Delta \rrbracket_{E}$. By construction, $\boldsymbol{\nabla}^{\xi} \Delta$ is a section of the bundle $\xi^{*} \otimes E$. Under the action of $\mathrm{SO}(2)$ the bundle $\xi^{*} \otimes E$ splits into two rank-2 subbundles, $F^{+} \oplus F^{-}$, where $F^{+}$is the spin-1 bundle corresponding to $+1 / 2$ profiles and $F^{-}$is the spin- 3 bundle corresponding to $-1 / 2$ profiles, and we decompose $\nabla^{\xi} \Delta$ according to this splitting as

$$
\boldsymbol{\nabla}^{\xi} \Delta=\nabla^{\xi} \Delta^{+}+\nabla^{\xi} \Delta^{-}
$$

A short calculation shows that $\mathbf{n} \cdot \boldsymbol{\nabla} \times \boldsymbol{B}=$ $\left|\boldsymbol{\nabla}^{\xi} \Delta^{+}\right|^{2}-\left|\nabla^{\xi} \Delta^{-}\right|^{2}$, so that if $\left|\boldsymbol{\nabla}^{\xi} \Delta^{+}\right|>\left|\boldsymbol{\nabla}^{\xi} \Delta^{-}\right|$, the umbilic has a local $+1 / 2$ profile, while if the converse holds, the profile is of type $-1 / 2$.

This analysis allows us to give a description of the space of local profiles for a generic umbilic. Since $\left.\nabla^{\xi} \Delta\right|_{\mathcal{U}}$ is nonzero for a generic umbilic, but otherwise arbitrary, the space of local profiles has the homotopy type of the threesphere. However, there is more structure to it than that. The equality $\left|\boldsymbol{\nabla}^{\xi} \Delta^{+}\right|=\left|\nabla^{\xi} \Delta^{-}\right|$defines a Clifford torus separating the three-sphere into two solid tori corresponding to the different profiles. A natural constraint on an umbilic loop is for its profile to be of constant type, i.e., for $\mathbf{n} \cdot \boldsymbol{\nabla} \times \boldsymbol{B}$ to be of constant sign or the profile to stay within a single solid torus. We call an umbilic loop that satisfies this constraint transverse, as its tangent vector is always transverse to the planes of $\xi$. This is not an unphysical constraint; transverse umbilic lines are commonly found in various chiral structures-typical $\lambda$ lines in cholesterics have a local structure in which $\mathbf{n}$ is either parallel or antiparallel with the tangent vector to an umbilic. Figure 4 shows the transverse umbilic loop found in toron excitations in frustrated cholesterics $[30,32]$.

The condition of an umbilic being transverse therefore translates as one of the two profiles having a higher "weight" along the entire length of the umbilic. If this is the case, then we have $\left|\nabla^{\xi} \Delta^{ \pm}\right|^{2} \neq 0$, with the sign depending on whether the umbilic is positively or negatively transverse. Suppose, for concreteness, that the umbilic is positively transverse. Then, in a local trivialization, we will have $\boldsymbol{\nabla}^{\xi} \Delta^{+}=t_{1} \mathbf{f}_{1}^{+}+t_{2} \mathbf{f}_{2}^{+}$, with $t_{1}^{2}+t_{2}^{2} \neq 0$, and the space of positively transverse umbilics has the homotopy type of a circle. The variation of the profile around a closed, transverse umbilic loop confers another integer winding number. This number depends on how the basis vectors of the trivialization of $F^{+}$wind around the umbilic. The ambiguity can be removed by demanding that each of the basis vectors in the trivialization does not link with the umbilic. As we show in the next section, this winding number can be interpreted as twice a self-linking number for the umbilic loop, and is related to a relative Euler class of $E$.

\section{UMBILIC LOOPS}

The umbilics of three-dimensional orientationally ordered materials are extended linelike objects; however, most of the properties we have described so far can be associated to isolated points along these one-dimensional curves. For example, the points of transverse intersection with an appropriate surface (or slice through the material) count numbers of Skyrmions, merons, or $\lambda$ defects. It is clear, however, that these locally measurable objects do not represent all of the information encoded by umbilic lines; if one has a closed umbilic loop, then, as discussed above, the local properties at each point must stitch together 
consistently along the loop conveying both geometric and topological information about the orientational order.

Umbilic loops have been observed in experimental systems $[3,32,44]$, and a motivating set of examples is the so-called torons [30], illustrated in Fig. 4. These soliton structures can be generated in a frustrated cholesteric cell, with the director held vertical along the top and bottom. As shown in Fig. 4(a), they display a characteristic umbilic loop, in the form of a circle sitting in the midplane of the cell. This loop is of generic type, with the eigenvectors of $\Pi$ having a monstar profile.

Indeed, one can show [118] that the existence of this umbilic loop is a consequence of the symmetries of the particular configuration. Consider the right-hand half of Fig. 4(c). Restricted to a path from the top to the bottom of the cell, to the right of the two hedgehogs, $\mathbf{n}$ traces out a great circle on the target $S^{2}$. As such, the right-hand half of Fig. 4(c) must cover a hemisphere on the target $S^{2}$. By symmetry, the other half of the image must cover the other hemisphere. By standard surgery operations, one can then stitch these two pieces together to create a map $S^{2} \rightarrow S^{2}$, which must be of odd degree. As a consequence, there must be axisymmetric umbilic lines present in the system. These can either be pushed to infinity or be of finite size. In the case of the toron, one can observe the umbilic loop in the center, along with the winding of the eigenvectors of $\Pi$ around the boundary.

As with local properties of umbilic lines, much of the structure of loops is encoded in the 1-form A. Previously, we obtained local information about umbilics by integrating $A$ around a meridian. The first step to understanding their global structure is to integrate $A$ along a longitude. Since $A$ is singular on an umbilic, we must define this integral as a limit. If $U_{i}$ is an umbilic, then let $U_{i}^{\prime}$ be a curve close to $U_{i}$ that has zero linking number with it. The integral of $A$ over $U_{i}^{\prime}$ is well defined, and if all the umbilics in the system are closed loops, we can take the limit $U_{i}^{\prime} \rightarrow U_{i}$ to obtain

$$
\int_{U_{i}} A=2 \pi \sum_{j} s_{j} \operatorname{Lk}\left(U_{i}, U_{j}\right)+\int_{\Sigma} \Omega_{E}
$$

where $\Sigma$ is an orientable surface bounded by $U_{i}$. The requirement that $U_{i}^{\prime}$ have zero linking with $U_{i}$ ensures that the limit is well defined, but in general the result depends upon this choice of framing. The quantity $\sum_{j} s_{j} \operatorname{Lk}\left(U_{i}, U_{j}\right)=e\left(U_{i}\right)$ in Eq. (28) is an integer associated to the umbilic line, the relative Euler class, and counts the number of umbilics linking $U_{i}$. There are two natural questions to ask of this quantity. The first is whether it corresponds to any local geometric structure of the umbilic line, the second is whether it relates to a global property of $\mathbf{n}$. On both counts the answer is yes. Geometrically, Eq. (28) is related to both a self-linking number and the winding number defined in the previous section. Globally, we show that it is related to the Hopf invariant of $\mathbf{n}$.

To see this, suppose $U$ is a transverse umbilic loop, then let $\hat{U}$ be a loop close to $U$ of zero linking number with $U$. Evaluate one of the eigenvectors of $\Delta, \mathbf{p}_{\Delta}^{+}$, say, along $\hat{U}$ and then push $U$ along this eigenvector field to form a new loop, $U^{\prime}$. The linking number $\operatorname{Lk}\left(U, U^{\prime}\right)$ is then taken to define the self-linking number of the transverse umbilic, $\operatorname{Sl}(U)$. This construction is shown in Fig. 8. Note that since $\mathbf{p}_{\Delta}^{+}$is a line field, $\mathrm{Sl}(U)$ may be a half-integer corresponding to $\mathbf{p}$ being nonorientable along the longitude of $U$.

With this notion in hand, the correspondence between the self-linking number and the winding number coming from the variation of the local profile defined in the previous section can be established. Choose a trivialization $\left\{\mathbf{d}_{1}, \mathbf{d}_{2}\right\}$ of $\xi$ on a neighborhood of $U$ such that the linking number of $U$ pushed off along $\mathbf{d}_{1}$ (say) is zero, then the winding of $\boldsymbol{\nabla}^{\xi} \Delta^{+}$with respect to the chosen trivialization will give twice the self-linking number. Note that the geometric definition of the self-linking number did not depend on the zero of $\Delta$ being of linear order. The relationship between the self-linking number and the relative Euler class of a transverse umbilic is then [119]

$$
\mathrm{Sl}(U)=\overline{\mathrm{Sl}}(U)+\frac{1}{2} e(U)
$$

There is a new term in this equation, $\overline{\mathrm{Sl}}(U)$, the transverse self-linking number of $U \cdot \overline{\mathrm{Sl}}(U)$ is a geometric quantity, preserved under deformations that keep $U$ transverse. A similar notion is studied in the context of contact topology [120], where it provides useful geometric information, but we do not know of a general discussion in the case of arbitrary $\mathbf{n}$.

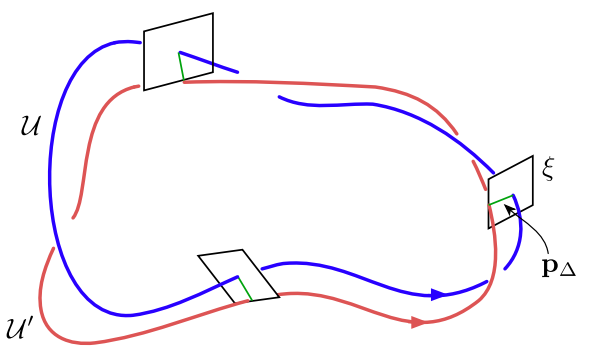

FIG. 8. The self-linking number for transverse umbilic loops. The red curve $U^{\prime}$ is formed by pushing $U$ along an eigenvector field $\mathbf{p}_{\Delta}$ of $\Delta$. The self-linking number is then computed as the linking number of $U$ with $U^{\prime}$. The umbilic must be transverse to ensure that $U^{\prime}$ does not cross $U$. Because the eigenvectors are degenerate on the umbilic, one evaluates $\mathbf{p}_{\Delta}$ on a line close to $U$, $\hat{U}$, that has zero self-linking number with $U$. Such a line can be found by drawing an orientable surface whose boundary is $U$ and pushing $U$ into that surface. Note that, because eigenvectors are line fields, the self-linking number can be a half-integer, corresponding to $\mathbf{p}$ being nonorientable along the longitude of $U$. 
We now turn to the relationship between the linking of umbilics and global properties of $\mathbf{n}$. Associated to the 1 -form $A$ is the Chern-Simons 3-form, $A \wedge d A$, and it is natural to ask whether the integral over the complement of $\mathcal{U}$,

$$
\int A \wedge d A
$$

gives some information about umbilic lines and $\mathbf{n}$. This integral can be compared to the similar helicity integral for a fluid flow and the Abelian Chern-Simons action. Both these quantities are related to linking numbers, of vortex lines in the case of helicity [53-55] and Wilson loops in Chern-Simons theory $[121,122]$, and, as we show, it is no different for umbilics; Eq. (30) connects the linking numbers of the umbilic loops with the Hopf invariant of n. To proceed, we assume that the material domain is $S^{3}$, with no defects, and that the zero set of $\Delta$ is one dimensional. By a compactification, this assumption that the domain is $S^{3}$ is equivalent to examining a system in $\mathbb{R}^{3}$ for which $\lim _{x \rightarrow \infty} \mathbf{n}(x)=\mathbf{n}_{0}$, a constant. An example of such a system is illustrated by the Hopf fibration, which has been experimentally realized in frustrated cholesterics [32], illustrated in Fig. 9, and the aformentioned toron. In both these systems $\mathbf{n}$ is constant on the boundary of the cell. Because of this, $\Delta=0$ on the boundary, and one should consider these systems as having an umbilic line passing through the point "at infinity," which in Figs. 4 and 9 is just the boundary of the system.

Topologically, the configuration in Fig. 9 is unusual; it is nonsingular but not homotopically equivalent to a constant. The difference is that it has a nonzero Hopf invariant. This integer invariant distinguishes topologically distinct nonsingular textures in $\mathbb{R}^{3}$ which are constrained to tend to a constant at infinity and is typically visualized in terms of linking of preimages of two orientations using the Pontryagin-Thom construction [32]. In our setting, however, one can give a slight reinterpretation of the famous Whitehead integral formula [123], similar to that given by Arnold [55] to show that the Hopf invariant is also the Chern-Simons invariant of the bundle $\xi$. In our interpretation, if one takes a $\omega$ to be a connection form for $\xi$, then $d \omega$ is the curvature of $\xi$ and the Chern-Simons integral,

$$
\frac{1}{(4 \pi)^{2}} \int_{S^{3}} \omega \wedge d \omega
$$

computes the Hopf invariant of $\mathbf{n}$.

Now, we turn back to our integral [Eq. (30)]. As discussed, we assume the material domain is $S^{3}$ (or $\mathbb{R}^{3}$ with uniform boundary conditions). This means that we can give a global trivialization of $\xi$ with connection $\omega$ and our 1 -form $A$ can be written as $A=d \theta+2 \omega$. This allows us to write

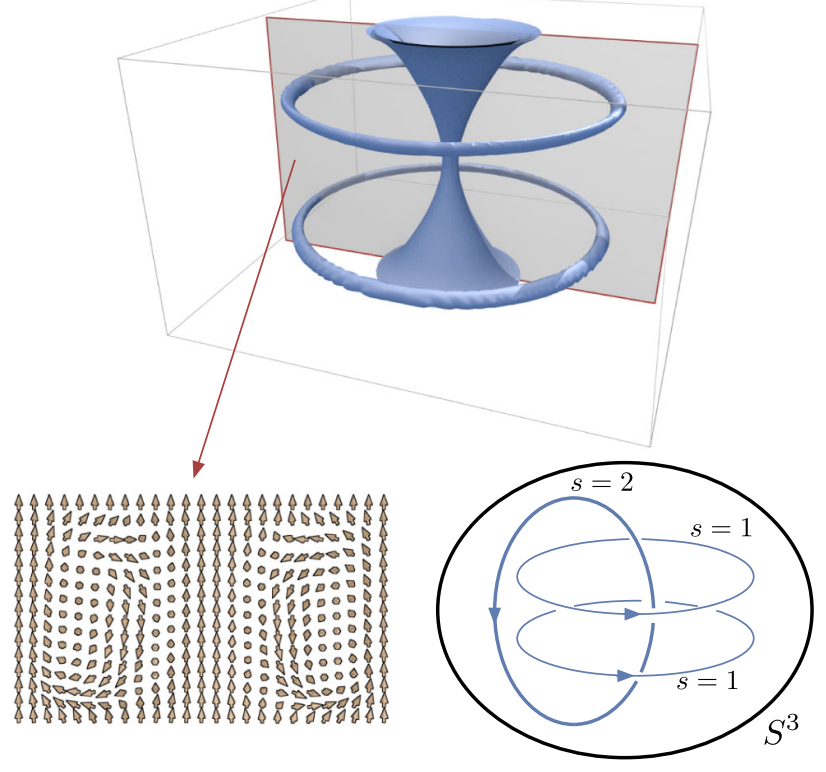

FIG. 9. Umbilic lines in the Hopf fibration. Top: Umbilic lines given as isosurfaces of $|\Delta|$ for a simulated Hopf fibration. The two lines in the midplane of the cell are both generic, with the same orientation. For symmetry reasons the central umbilic is not generic and has $s=2$. On the boundary of the cell $\mathbf{n}$ is uniform, which means that the Hopf invariant is well defined. Note that the system is totally umbilic on the boundary. If you compactify the domain by collapsing the boundary to a point, then one can interpret the central vertical umbilic line as passing through this point. Bottom left: Director field for the Hopf texture. Bottom right: Schematic of the relationship between umbilic lines in $S^{3}$. Two generic $s=1$ umbilic lines each link with one $s=2$ umbilic line that passes through the point at infinity.

$\int_{S^{3}-\mathcal{U}} A \wedge d A=4 \int_{S^{3}-\mathcal{U}} \omega \wedge d \omega+2 \int_{S^{3}-\mathcal{U}} d \theta \wedge d \omega$,

or, since $\mathcal{U}$ has measure zero,

$$
\int_{S^{3}-\mathcal{U}} A \wedge d A=4(4 \pi)^{2} H+2 \int_{S^{3}-\mathcal{U}} d \theta \wedge d \omega,
$$

where $H$ is the Hopf invariant of $\mathbf{n}$. Now, we need to evaluate the integral of $d \theta \wedge d \omega$ over $S^{3}-\mathcal{U}$. We first do a simpler integral, over $S^{3}-N(\mathcal{U})$, where $N(\mathcal{U})$ is a neighborhood of the umbilic lines. By Stokes's theorem this can be reduced to an integral over the boundary of $N(\mathcal{U})$ as

$$
\int_{S^{3}-N(\mathcal{U})} d \theta \wedge d \omega=\sum_{i} \int_{\partial N\left(U_{i}\right)} \omega \wedge d \theta
$$

The boundary of the neighborhood of each umbilic line is a torus, and because our domain is $S^{3}$, we can choose a preferred longitude for each torus that has zero linking with the umbilic at its center. This allows us to specify a decomposition of each boundary component into $S_{M}^{1} \times S_{L}^{1}$. 
If we choose $N(\mathcal{U})$ small enough, then along each meridian of the torus $\omega$ will be approximately constant and equal to its value on the umbilic line. The integral over each boundary component can then be written as

$$
\sum_{i} \int_{\partial N\left(U_{i}\right)} \omega \wedge d \theta=\sum_{i} \int_{S_{L}^{1}} \omega^{*}\left(\int_{S_{M}^{1}} d \theta^{*}\right)
$$

where we have pulled the forms back to each $S^{1}$.

The integral of $d \theta$ is trivial; it evaluates to $s_{i} 2 \pi$, where $s_{i}$ is the strength of the $i$ th umbilic. Finally, we take a limit as $N(\mathcal{U}) \rightarrow \mathcal{U}$ and obtain a sum of Wilson loop integrals:

$$
\lim _{N(\mathcal{U}) \rightarrow \mathcal{U}} \sum_{i} \int_{\partial N\left(U_{i}\right)} \omega \wedge d \theta=2 \pi \sum_{i} s_{i} \int_{U_{i}} \omega .
$$

Now, from the Gauss-Bonnet-Chern theorem [Eq. (20)] we can relate intergals of $\omega$ along umbilics to integrals of $A$. Putting this together with the above equations, we obtain

$$
\begin{gathered}
\frac{1}{(4 \pi)^{2}} \int_{S^{3}-\mathcal{U}} A \wedge d A-\frac{1}{8 \pi} \sum_{i} s_{i} \int_{U_{i}} A \\
=4 H-\frac{1}{4} \sum_{i j} s_{i} s_{j} \operatorname{Lk}\left(U_{i}, U_{j}\right) .
\end{gathered}
$$

This equation relates analytic data, the integral of $A \wedge d A$ and Wilson loop integrals, to topological data, namely, the linking number of the umbilic lines and the Hopf invariant. We hope that this relationship will allow for an interpretation of the creation and destruction of Hopf solitons in terms of umbilic lines.

\section{SUMMARY}

Historically, the topology of ordered materials has been understood using homotopy theory. Umbilic lines, which arise naturally from the geometry of orientational order, provide an alternative framework in which to understand the structure of such systems. This approach has a distinct advantage over the traditional homotopy theoretic approach, as it is inherently associated with the geometric structure of the system. In this way, it provides a precise and mathematically rigorous way to determine the location of Skyrmions in a given system, particularly in three dimensions where they are realized as lines. In addition, it allows one to identify a number of other features in orientational order, such as $\lambda$ lines, double twist cylinders, and merons.

Locally, the structure of umbilic lines has analogies with many other areas of physics, including $C$ lines of polarization, helicity in fluid mechanics, and the chirality pseudotensor introduced recently by Efrati and Irvine in their study of chirality in materials [78]. This local structure reveals the difference between the profile of the umbilic and its orientation, illustrated in Eq. (26), that does not appear in the geometry of surfaces.

Globally, umbilic lines must satisfy a constraint coming from the Euler class of the bundle $E$. This global constraint allows one to identify the topology of systems through their umbilic lines, even when the topological class of the system is torsion. In addition to identifying Skyrmions in a system, this constraint allows one to understand the global structure of $\lambda$ lines, merons, and other similar objects. More subtly, umbilic loops can twist and link, with a number of phenomena appearing, including self-linking numbers and connections to Chern-Simons theory, helicity, and the Hopf invariant. These theoretical constructions are natural geometrically, but the experimental systems where they make an appearance are currently limited in number. We hope that our work will inspire future studies exploring these phenomena.

\section{ACKNOWLEDGMENTS}

We thank Mark Dennis, Randy Kamien, Dan Beller, Simon Čopar, Ricardo Mosna, Daniel Sussman, and Antoine Rémond-Tiedrez for useful and invigorating discussions. This work was partially supported by the UK EPSRC through Grants No. A.MACX.0002 (T. M. and G. P. A.) and No. EP/N007883/1 (G. P. A.). T. M. was also partially supported by a University of Warwick Chancellor's International Scholarship and a University of Warwick IAS Early Career Fellowship.

[1] R. B. Meyer, On the Existence of Even Indexed Disclinations in Nematic Liquid Crystals, Philos. Mag. 27, 405 (1973).

[2] C. Williams and Y. Bouligand, Fils et Disinclinaisons dans un Nématique en Tube Capillaire, J. Phys. (Paris) 35, 589 (1974).

[3] P. J. Ackerman, R. P. Trivedi, B. Senyuk, J. van de Lagemaat, and I. I. Smalyukh, Two-Dimensional Skyrmions and Other Solitonic Structures in ConfinementFrustrated Chiral Nematics, Phys. Rev. E 90, 012505 (2014).

[4] M. Kléman and J. Friedel, Lignes de Dislocation dans les Cholestériques, J. Phys. (Paris), Colloq. 30, C4-43 (1969).

[5] Y. Bouligand, Recherches sur les Textures des Etats Mésomorphes. 6 Dislocations Coins et Signification des Cloisons de Grandjean-Cano dans les Cholestériques, J. Phys. II (France) 35, 959 (1974).

[6] Y. Bouligand, B. Derrida, V. Poénaru, Y. Pomeau, and G. Toulouse, Distortions with Double Topological Character: The Case of Cholesterics, J. Phys. II (France) 39, 863 (1978).

[7] I. I. Smalyukh and O. D. Lavrentovich, Three-Dimensional Director Structures of Defects in Grandjean-Cano Wedges of Cholesteric Liquid Crystals Studied by Fluorescence Confocal Polarizing Microscopy, Phys. Rev. E 66, 051703 (2002). 
[8] S. Meiboom, J. P. Sethna, P. W. Anderson, and W. F. Brinkman, Theory of the Blue Phase of Cholesteric Liquid Crystals, Phys. Rev. Lett. 46, 1216 (1981).

[9] D. C. Wright and N. D. Mermin, Crystalline Liquids: The Blue Phases, Rev. Mod. Phys. 61, 385 (1989).

[10] N. D. Mermin and T. L. Ho, Circulation and Angular Momentum in the A Phase of Superfluid Helium-3, Phys. Rev. Lett. 36, 594 (1976).

[11] P. W. Anderson and G. Toulouse, Phase Slippage without Vortex Cores: Vortex Textures in Superfluid ${ }^{3} \mathrm{He}$, Phys. Rev. Lett. 38, 508 (1977).

[12] G. E. Volovik and V. P. Mineev, Vortices with Free Ends in Superfluid $\mathrm{He}^{3}-A$, Pis'ma Zh. Eksp. Teor. Fiz. 23, 647 (1976) [JETP Lett. 23, 593 (1976)].

[13] P. M. Walmsley and A. I. Golov, Chirality of Superfluid ${ }^{3}$ He-A, Phys. Rev. Lett. 109, 215301 (2012).

[14] We use the word "Skyrmion" in the context of textures observed in chiral magnets, rather than in its original context of a nonlinear field theory of pions. This duplicity seems to be sufficiently well established, and widely known, so that the potential for confusion is minimal.

[15] X. Yu, M. Mostovoy, Y. Tokunaga, W. Zhang, K. Kimoto, Y. Matsui, Y. Kaneko, N. Nagaosa, and Y. Tokura, Magnetic Stripes and Skyrmions with Helicity Reversals, Proc. Natl. Acad. Sci. U.S.A. 109, 8856 (2012).

[16] P. Milde, D. Köhler, J. Seidel, L. M. Eng, A. Bauer, A. Chacon, J. Kindervater, S. Mühlbauer, C. Pfleiderer, S. Buhrandt, C. Schütte, and A. Rosch, Unwinding of a Skyrmion Lattice by Magnetic Monopoles, Science 340, 1076 (2013).

[17] S. Mühlbauer, B. Binz, F. Jonietz, C. Pfleiderer, A. Rosch, A. Neubauer, R. Georgii, and P. Böni, Skyrmion Lattice in a Chiral Magnet, Science 323, 915 (2009).

[18] U. K. Rößler, A. N. Bogdanov, and C. Pfleiderer, Spontaneous Skyrmion Ground States in Magnetic Metals, Nature (London) 442, 797 (2006).

[19] X. Z. Yu, Y. Onose, N. Kanazawa, J. H. Park, J. H. Han, Y. Matsui, N. Nagaosa, and Y. Tokura, Real-Space Observation of a Two-Dimensional Skyrmion Crystal, Nature (London) 465, 901 (2010).

[20] A. N. Bogdanov and D. A. Yablonskii, Thermodynamically Stable "Vortices" in Magnetically Ordered Crystals. The Mixed State of Magnets, Sov. Phys. JETP 68, 101 (1989).

[21] A. N. Bogdanov and A. Hubert, Thermodynamically Stable Magnetic Vortex States in Magnetic Crystals, J. Magn. Magn. Mater. 138, 255 (1994).

[22] A. N. Bogdanov, New Localized Solutions of the Nonlinear Field Equations, JETP Lett. 62, 247 (1995).

[23] L. S. Leslie, A. Hansen, K. C. Wright, B. M. Deutsch, and N. P. Bigelow, Creation and Detection of Skyrmions in a Bose-Einstein Condensate, Phys. Rev. Lett. 103, 250401 (2009).

[24] M. J. H. Ku, W. Ji, B. Mukherjee, E. Guardado-Sanchez, L. W. Cheuk, T. Yefsah, and M. W. Zwierlein, Motion of a Solitonic Vortex in the BEC-BCS Crossover, Phys. Rev. Lett. 113, 065301 (2014).

[25] S. Donadello, S. Serafini, M. Tylutki, L. P. Pitaevskii, F. Dalfovo, G. Lamporesi, and G. Ferrari, Observation of
Solitonic Vortices in Bose-Einstein Condensates, Phys. Rev. Lett. 113, 065302 (2014).

[26] U. Al Khawaja and H. Stoof, Skyrmions in a Ferromagnetic Bose-Einstein Condensate, Nature (London) 411, 918 (2001).

[27] J. Y. Choi, W. J. Kwon, and Y. I. Shin, Observation of Topologically Stable 2D Skyrmions in an Antiferromagnetic Spinor Bose-Einstein Condensate, Phys. Rev. Lett. 108, 035301 (2012).

[28] J. Y. Choi, S. Kang, S. W. Seo, W. J. Kwon, and Y. I. Shin, Observation of a Geometric Hall Effect in a Spinor BoseEinstein Condensate with a Skyrmion Spin Texture, Phys. Rev. Lett. 111, 245301 (2013).

[29] J. I. Fukuda and S. Žumer, Quasi-Two-Dimensional Skyrmion Lattices in a Chiral Nematic Liquid Crystal, Nat. Commun. 2, 246 (2010).

[30] I. I. Smalyukh, Y. Lansac, N. A. Clark, and R. P. Trivedi, Three-Dimensional Structure and Multistable Optical Switching of Triple-Twisted Particle-like Excitations in Anisotropic Fluids, Nat. Mater. 9, 139 (2010).

[31] P. J. Ackerman, Z. Qi, and I. I. Smalyukh, Optical Generation of Crystalline, Quasicrystalline, and Arbitrary Arrays of Torons in Confined Cholesteric Liquid Crystals for Patterning of Optical Vortices in Laser Beams, Phys. Rev. E 86, 021703 (2012).

[32] Bryan Gin-ge Chen, P. J. Ackerman, G. P. Alexander, R. D. Kamien, and I. I. Smalyukh, Generating the Hopf Fibration Experimentally in Nematic Liquid Crystals, Phys. Rev. Lett. 110, 237801 (2013).

[33] M. B. Pandey, T. Porenta, J. Brewer, A. Burkart, S. Čopar, S. Žumer, and I. I. Smalyukh, Self-Assembly of SkyrmionDressed Chiral Nematic Colloids with Tangential Anchoring, Phys. Rev. E 89, 060502 (2014).

[34] J. A. Martínez-González, Y. Zhou, M. Rahimi, E. Bukusoglu, N. L. Abbott, and J. J. de Pablo, Blue-Phase Liquid Crystal Droplets, Proc. Natl. Acad. Sci. U.S.A. 112, 13195 (2015).

[35] U. Tkalec, M. Ravnik, S. Žumer, and I. Muševič, Vortexlike Topological Defects in Nematic Colloids: Chiral Colloidal Dimers and 2D Crystals, Phys. Rev. Lett. 103, 127801 (2009).

[36] D. A. Beller, T. Machon, S. Čopar, D. M. Sussman, G. P. Alexander, R. D. Kamien, and R. A. Mosna, Geometry of the Cholesteric Phase, Phys. Rev. X 4, 031050 (2014).

[37] A. A. Belavin and A. M. Polyakov, Metastable States of Two-Dimensional Isotropic Ferromagnets Pis'ma Zh. Eksp. Teor. Fiz. 22, 503 (1975) [JETP Lett. 22, 245 (1975)].

[38] J. F. Nye, Lines of Circular Polarization in Electromagnetic Wave Fields, Proc. R. Soc. A 389, 279 (1983).

[39] J. F. Nye and J. V. Hajnal, The Wave Structure of Monochromatic Electromagnetic Radiation, Proc. R. Soc. A 409, 21 (1987).

[40] J. V. Hajnal, Singularities in the Transverse Fields of Electromagnetic Waves. I. Theory, Proc. R. Soc. A 414, 433 (1987).

[41] J. V. Hajnal, Singularities in the Transverse Fields of Electromagnetic Waves. II. Observations on the Electric Field, Proc. R. Soc. A 414, 447 (1987). 
[42] M. V. Berry and M. R. Dennis, Polarization Singularities in Isotropic Random Waves, Proc. R. Soc. A 456, 2059 (2000).

[43] M. V. Berry and M. R. Dennis, Polarization Singularities in Isotropic Random Vector Waves, Proc. R. Soc. A 457, 141 (2001).

[44] F. Flossmann, K. O’Holleran, M. R. Dennis, and M. J. Padgett, Polarization Singularities in $2 D$ and $3 D$ Speckle Fields, Phys. Rev. Lett. 100, 203902 (2008).

[45] M. Ezawa, Compact Merons and Skyrmions in Thin Chiral Magnetic Films, Phys. Rev. B 83, 100408(R) (2011).

[46] M. R. Dennis, R. P. King, B. Jack, K. O'Holleran, and M. J. Padgett, Isolated Optical Vortex Knots, Nat. Phys. 6, 118 (2010).

[47] U. Tkalec, M. Ravnik, S. Čopar, S. Žumer, and I. Muševič, Reconfigurable Knots and Links in Chiral Nematic Colloids, Science 333, 62 (2011).

[48] D. Kleckner and W. T. M. Irvine, Creation and Dynamics of Knotted Vortices, Nat. Phys. 9, 253 (2013).

[49] T. Machon and G. P. Alexander, Knots and Nonorientable Surfaces in Chiral Nematics, Proc. Natl. Acad. Sci. U.S.A. 110, 14174 (2013).

[50] A. Martinez, M. Ravnik, B. Lucero, R. Visvanathan, S. Žumer, and I. I. Smalyukh, Mutually Tangled Colloidal Knots and Induced Defect Loops in Nematic Fields, Nat. Mater. 13, 258 (2014).

[51] M. Tasinkevych, M. G. Campbell, and I. I. Smalyukh, Splitting, Linking, Knotting, and Solitonic Escape of Topological Defects in Nematic Drops with Handles, Proc. Natl. Acad. Sci. U.S.A. 111, 16268 (2014).

[52] T. Machon and G. P. Alexander, Knotted Defects in Nematic Liquid Crystals, Phys. Rev. Lett. 113, 027801 (2014).

[53] H. K. Moffatt, The Degree of Knottedness of Tangled Vortex Lines, J. Fluid Mech. 35, 117 (1969).

[54] H. K. Moffatt and R. L. Ricca, Helicity and the Călugăreanu Invariant, Proc. R. Soc. A 439, 411 (1992).

[55] V. I. Arnold, in A. B. Givental, B. A. Khesin, A. N. Varchenko, V. A. Vassiliev, and O. Ya. Viro, V.I. Arnold - Collected Works (Springer, 2014), pp. 357-375.

[56] O. D. Lavrentovich, Liquid Crystals, Photonic Crystals, Metamaterials, and Transformation Optics, Proc. Natl. Acad. Sci. U.S.A. 108, 5143 (2011).

[57] O. D. Lavrentovich, Designing Dupin Cyclides in Micro and Macro Worlds, Proc. Natl. Acad. Sci. U.S.A. 110, 5 (2013).

[58] I. Muševič and S. Žumer, Liquid Crystals: Maximizing Memory, Nat. Mater. 10, 266 (2011).

[59] I. Muševič, Nematic Colloids, Topology and Photonics, Phil. Trans. R. Soc. A 371, 20120266 (2013).

[60] A. Fert, V. Cros, and J. Sampaio, Skyrmions on the Track, Nat. Nanotechnol. 8, 152 (2013).

[61] N. S. Kiselev, A. N. Bogdanov, R. Schäfer, and U. K. Rößler, Chiral Skyrmions in Thin Magnetic Films: New Objects for Magnetic Storage Technologies?, J. Phys. D 44, 392001 (2011).

[62] R. Duine, Spintronics: Skyrmions Singled Out, Nat. Nanotechnol. 8, 800 (2013).

[63] D. Hilbert and S. Cohn-Vossen, Geometry and the Imagination (AMS Chelsea Publishing, New York, 1999).
[64] M. V. Berry and J. H. Hannay, Umbilic Points on Gaussian Random Surfaces, J. Phys. A 10, 1809 (1977).

[65] M. R. Dennis, Polarization Singularities in Paraxial Vector Fields: Morphology and Statistics, Opt. Commun. 213, 201 (2002).

[66] F. Flossmann, U. T. Schwarz, M. Maier, and M. R. Dennis, Polarization Singularities from Unfolding an Optical Vortex through a Birefringent Crystal, Phys. Rev. Lett. 95, 253901 (2005).

[67] V. Vitelli, B. Jain, and R. D. Kamien, Topological Defects in Gravitational Lensing Shear Fields, J. Cosmol. Astropart. Phys. 09 (2009) 034.

[68] T. H. Beuman, A. M. Turner, and V. Vitelli, Stochastic Geometry and Topology of Non-Gaussian Fields, Proc. Natl. Acad. Sci. U.S.A. 109, 19943 (2012).

[69] T. H. Beuman, A. M. Turner, and V. Vitelli, Critical and Umbilical Points of a Non-Gaussian Random Field, Phys. Rev. E 88, 012115 (2013).

[70] M. V. Berry, Quantal Phase Factors Accompanying Adiabatic Changes, Proc. R. Soc. A 392, 45 (1984).

[71] M. V. Berry, in Geometric Phases in Physics, edited by A. Shapere and F. Wilczek (World Scientific, Singapore, 1989).

[72] A. Neubauer, C. Pfleiderer, B. Binz, A. Rosch, R. Ritz, P. G. Niklowitz, and P. Böni, Topological Hall Effect in the A Phase of MnSi, Phys. Rev. Lett. 102, 186602 (2009).

[73] K. Everschor, M. Garst, R. A. Duine, and A. Rosch, Current-Induced Rotational Torques in the Skyrmion Lattice Phase of Chiral Magnets, Phys. Rev. B 84, 064401 (2011).

[74] T. Schultz, R. Ritz, A. Bauer, M. Halder, M. Wagner, C. Franz, C. Pfleiderer, K. Everschor, M. Garst, and A. Rosch, Emergent Electrodynamics of Skyrmions in a Chiral Magnet, Nat. Phys. 8, 301 (2012).

[75] P. G. de Gennes and J. Prost, The Physics of Liquid Crystals, 2nd ed. (Oxford University Press, Oxford, 1993).

[76] I. Dzyaloshinsky, A Thermodynamic Theory of "Weak" Ferromagnetism of Antiferromagnetics, J. Phys. Chem. Solids 4, 241 (1958).

[77] T. Moriya, Anisotropic Superexchange Interaction and Weak Ferromagnetism, Phys. Rev. 120, 91 (1960).

[78] E. Efrati and W. T. M. Irvine, Orientation-Dependent Handedness and Chiral Design, Phys. Rev. X 4, 011003 (2014).

[79] A. S. Thorndike, C. R. Cooley, and J. F. Nye, The Structure and Evolution of Flow Fields and Other Vector Fields, J. Phys. A 11, 1455 (1978).

[80] S. Čopar, M. R. Dennis, R. D. Kamien, and S. Žumer, Singular Values, Nematic Disclinations, and Emergent Biaxiality, Phys. Rev. E 87, 050504(R) (2013).

[81] S. Banerjee, J. Rowland, O. Erten, and M. Randeria, Enhanced Stability of Skyrmions in Two-Dimensional Chiral Magnets with Rashba Spin-Orbit Coupling, Phys. Rev. X 4, 031045 (2014).

[82] R. B. Meyer, Piezoelectric Effects in Liquid Crystals, Phys. Rev. Lett. 22, 918 (1969).

[83] R. A. P. Rogers, Some Differential Properties of the Orthogonal Trajectories of a Congruence of Curves, with an Application to Curl and Divergence of Vectors, Proc. R. Irish Soc. A 29, 92 (1912). 
[84] Yu. Aminov, The Geometry of Vector Fields (Gordon and Breach Science Publishers, Amsterdam, 2000).

[85] N. S. Manton and P. M. Sutcliffe, Topological Solitons (Cambridge University Press, Cambridge, England, 2004).

[86] S. Armon, E. Efrati, R. Kupferman, and E. Sharon, Geometry and Mechanics in the Opening of Chiral Seed Pods, Science 333, 1726 (2011).

[87] G. P. Alexander, B. G. Chen, E. A. Matsumoto, and R. D. Kamien, Colloquium: Disclination Loops, Point Defects, and All That in Nematic Liquid Crystals, Rev. Mod. Phys. 84, 497 (2012).

[88] N. D. Mermin, The Topological Theory of Defects in Ordered Media, Rev. Mod. Phys. 51, 591 (1979).

[89] S. E. Korshunov, Phase Diagram of the Modified XY Model, J. Phys. C 19, 4427 (1986).

[90] A. J. A. James and A. Lamacraft, Phase Diagram of Two-Dimensional Polar Condensates in a Magnetic Field, Phys. Rev. Lett. 106, 140402 (2011).

[91] H. Grebel, R. M. Hornreich, and S. Shtrikman, Landau Theory of Cholesteric Blue Phases, Phys. Rev. A 28, 1114 (1983).

[92] P. Pieranski, P.E. Cladis, and R. Barbet-Massin, Experimental Evidence for a Hexagonal Blue Phase, J. Phys. (Paris), Lett. 46, 973 (1985).

[93] P. Pieranski and P. E. Cladis, Field-Induced Tetragonal Blue Phase (BP X), Phys. Rev. A 35, 355 (1987).

[94] R. M. Hornreich and S. Shtrikman, Field-Induced Hexagonal Blue Phases in Positive and Negative Dielectric Anisotropy Systems: Phase Diagrams and Topological Properties, Phys. Rev. A 41, 1978 (1990).

[95] J. Fukuda and S. Žumer, Cholesteric Blue Phases: Effect of Strong Confinement, Liq. Cryst. 37, 875 (2010).

[96] O. Henrich, K. Stratford, M. E. Cates, and D. Marenduzzo, Structure of Blue Phase III of Cholesteric Liquid Crystals, Phys. Rev. Lett. 106, 107801 (2011).

[97] B. Binz, A. Vishwanath, and V. Aji, Theory of the Helical Spin Crystal: A Candidate for the Partially Ordered State of MnSi, Phys. Rev. Lett. 96, 207202 (2006).

[98] A. Hamann, D. Lamago, Th. Wolf, H. v. Löhneysen, and D. Reznik, Magnetic Blue Phase in the Chiral Itinerant Magnet MnSi, Phys. Rev. Lett. 107, 037207 (2011).

[99] A. O. Leonov, I. E. Dragunov, U. K. Rößler, and A. N. Bogdanov, Theory of Skyrmion States in Liquid Crystals, Phys. Rev. E 90, 042502 (2014).

[100] We are reminded here of a sentiment put forth by Michael Atiyah: "Algebra is the offer made by the devil to the mathematician. The devil says: 'I will give you this powerful machine, it will answer any question you like. All you need to do is give me your soul: give up geometry and you will have this marvellous machine."” M. F. Atiyah, Mathematics in the 20th Century, Bull. London Math. Soc. 34, 1 (2002).

[101] R. M. Hornreich and S. Shtrikman, Topological Properties of Cholesteric Blue Phases, Phys. Rev. A 38, 4843 (1988).

[102] G. P. Alexander, D.Phil. thesis, University of Oxford, 2008.
[103] M. Ravnik, G. P. Alexander, J. M. Yeomans, and S. Žumer, Three-Dimensional Colloidal Crystals in Liquid Crystalline Blue Phases, Proc. Natl. Acad. Sci. U.S.A. 108, 5188 (2011).

[104] T. Machon, Ph.D. thesis, University of Warwick, 2015.

[105] R. Bott and L. W. Tu, Differential Forms in Algebraic Topology (Springer-Verlag, New York, 1982).

[106] J. W. Milnor and J. D. Stasheff, Characteristic Classes (Princeton University Press, Princeton, 1974).

[107] P. A. M. Dirac, Quantised Singularities in the Electromagnetic Field, Proc. R. Soc. A 133, 60 (1931).

[108] S. S. Chern, A Simple Intrinsic Proof of the Gauss-Bonnet Formula for Closed Riemannian Manifolds, Ann. Math. 45, 747 (1944).

[109] S.S. Chern, On the Curvatura Integra in a Riemannian Manifold, Ann. Math. 46, 674 (1945).

[110] S.S. Chern, Characteristic Classes of Hermitian Manifolds, Ann. Math. 47, 85 (1946).

[111] S. Seki, X. Z. Yu, S. Ishiwata, and Y. Tokura, Observation of Skyrmions in a Multferroic Material, Science 336, 198 (2012).

[112] X. Z. Yu, N. Kanazawa, Y. Onose, K. Kimoto, W.Z. Zhang, S. Ishiwata, Y. Matsui, and Y. Tokura, Near Room-Temperature Formation of a Skyrmion Crystal in Thin-Films of Helimagnet FeGe, Nat. Mater. 10, 106 (2011).

[113] A. Tonomura, X. Yu, K. Yanagisawa, T. Matsuda, Y. Onose, N. Kanazawa, H. S. Park, and Y. Tokura, RealSpace Observation of Skyrmion Lattice in Helimagnet MnSi Thin Samples, Nano Lett. 12, 1673 (2012).

[114] R. D. Kamien, Geometry of Soft Materials: A Primer, Rev. Mod. Phys. 74, 953 (2002).

[115] If $\mathbf{n}$ is considered as a map into $S^{2}$, then homotopy classes are given by an element in $H^{2}$ with additional data associated to the Hopf invariant.

[116] Here, torsion is meant in the sense of group theory-i.e., $H^{2}(M)$ contains elements of finite order-and not in the geometric sense.

[117] M. V. Berry, Index Formulae for Singular Lines of Polarization, J. Opt. A 6, 675 (2004).

[118] For a more detailed version of this argument, readers are invited to contact the authors.

[119] To see that Eq. (29) is correct, it is enough to show that increasing $e(U)$ by 1 increases $\operatorname{Sl}(U)$ by $1 / 2$ coupled with standard arguments from contact topology [120].

[120] H. Geiges, An Introduction to Contact Topology (Cambridge University Press, Cambridge, England, 2008).

[121] A. M. Polyakov, One Physical Problem with Possible Mathematical Significance, J. Geom. Phys. 5, 595 (1988).

[122] E. Witten, Quantum Field Theory and the Jones Polynomial, Commun. Math. Phys. 121, 351 (1989).

[123] J. H. C. Whitehead, An Expression of Hopf's Invariant as an Integral, Proc. Natl. Acad. Sci. U.S.A. 33, 117 (1947). 